Mon. Not. R. Astron. Soc. 000,1-?? (2016) ～Printed 2 June $2021 \quad$ (MN LATEX style file v2.2)

\title{
THE FREQUENCY OF BINARY STAR INTERLOPERS AMONGST TRANSITIONAL DISKS.
}

\author{
D. Ruíz-Rodríguez ${ }^{1}$, M. Ireland ${ }^{1}$, L. Cieza ${ }^{2,3}$, A. Kraus ${ }^{4}$ \\ ${ }^{1}$ Research School of Astronomy and Astrophysics, Australian National University, Canberra, ACT 2611, Australia; dary.ruiz@anu.edu.au \\ ${ }^{2}$ Núcleo de Astronomía, Facultad de Ingenería, Universidad Diego Portales, Av. Ejercito 441, Santiago, Chile \\ ${ }^{3}$ Millenium Nucleus "Protoplanetary Disks in ALMA Early Science", Chile \\ ${ }^{4}$ Department of Astronomy, The University of Texas at Austin, Austin, TX 78712, USA
}

\begin{abstract}
Using Non-Redundant Mask interferometry (NRM), we searched for binary companions to objects previously classified as Transitional Disks (TD). These objects are thought to be an evolutionary stage between an optically thick disk and optically thin disk. We investigate the presence of a stellar companion as a possible mechanism of material depletion in the inner region of these disks, which would rule out an ongoing planetary formation process in distances comparable to the binary separation. For our detection limits, we implement a new method of completeness correction using a combination of randomly sampled binary orbits and Bayesian inference. The selected sample of 24 TDs belong to the nearby and young star forming regions: Ophiuchus ( $130 \mathrm{pc})$, Taurus-Auriga $(\sim 140 \mathrm{pc})$ and IC348 $(\sim 220 \mathrm{pc})$. These regions are suitable to resolve faint stellar companions with moderate to high confidence levels at distances as low as 2 au from the central star. With a total of 31 objects, including 11 known TDs and circumbinary disks from the literature, we have found that a fraction of $0.38 \pm 0.09$ of the SEDs of these objects are likely due to the tidal interaction between a close binary and its disk, while the remaining SEDs are likely the result of other internal processes such as photoevaporation, grain growth, planet disk interactions. In addition, we detected four companions orbiting outside the area of the truncation radii and we propose that the IR excesses of these systems are due to a disk orbiting a secondary companion
\end{abstract}

Key words: Transitional Disks, Binary stars, Bayesian Analysis and Non-redundant Masking, Optical Interferometry.

\section{INTRODUCTION}

After the formation of a star, the lifetime of a disk is estimated to be $<10$ Myrs. At an age of $\sim 5$ Myrs, around $90 \%$ of these objects already went through an evolution process of dispersion of their optically thick primordial disks (Sicilia-Aguilar et al. 2006). The dispersion of the inner disk material creates unique morphologies in the disk that can be detected by their unusual spectral energy distributions (SED) (Strom et al. 1989). Assuming that all disks go through this dispersing phase, then approximately 10-20\% of the disks are in a "transition" phase with time-scales within $<0.5 \mathrm{Myr}$; (Furlan et al. 2011; Koepferl et al. 2013). In comparison with the characteristic continuum level of the SED of a Classical T Tauri Star (CTTS), these objects are defined as: stellar objects with small near-infrared (NIR) and/or mid-infrared (MIR) excesses and large MIR and/or far-infrared (FIR) excesses (e.g. Espaillat et al. 2014). Given the ambiguity in the literature as to whether a disk in a "transition phase" makes reference exclusively to a disk with an inner hole surrounding a single star or also includes binary systems in a transition phase, we will describe disks around single stars exclusively as Transitional Disk (TD) and to describe disks around binary stars as Circumbinary Disks (CD).

Detailed modelling of TD disk SEDs has interpreted the reduction of excess in the NIR-MIR as the dearth of small dust grains and thin gas in the inner region of the disk (Espaillat et al.2012). In addition, mm-interferometric observations have mapped this particular disk morphology of the TDs, showing a dust-depleted region in the inner disk and/or gaps (Andrews et al. 2011; Canovas et al. 2016). Although the physical origins causing these particular shapes in the disks are still unclear, several theories have been proposed to explain the clearing mechanisms in the disk from inside out, such as grain growth (Dullemond, Dominik \& Natta 2001), magnetorotational instability (Chiang \& Murray-Clay 2007), photoevaporation (Clarke. Gendrin \& Sotomavor 2001; Alexander \& Armitage 2007), dust filtration (Rice et al. 2006a), and disk-planet(s) interactions (Kraus et al. 2012: Dodson-Robinson \& Salyk 2011). However, it has been difficult to reconcile the main process of dispersion of the disk, especially since these mecha- 
nisms might dominate at different time-scales and radii. For instance, planet formation and photoevaporation may play a sequential dominating role in the disk dispersion phase, since photoevaporation disperses more rapidly once a planet is formed and has carved a gap in the disk (Rosotti, Ercolano \& Owen 2015).

Unfortunately, these models are still not able to simultaneously explain the evolution process of all TDs, especially those with high accretion rates and large inner cavities full of large amounts of gas near the central star. However, fully understanding the disk dispersal process is of a vital importance, because it provides insights about the formation of planetary systems like our own (DodsonRobinson \& Salyk 2011). In particular, knowledge of the timescales of gas survival sets constraints on the time available for the formation of a gas rich planet via core accretion (Pollack et al. 1996). Alternatively, another clearing mechanism has been proposed for the truncation of the inner disk: the presence of a stellar companion. Artymowicz \& Lubow (1994) showed that in the binary-disk interaction, the stellar companion will truncate the $\mathrm{CD}$ at a distance, which depends highly on the eccentricity and mass ratio of the binary system. These theoretical models predict that the ratio of the inner radii $\left(r_{d}\right)$ about the center of mass and the semi-major axis $(a)$ of the binary system ranges from 1.7 to 3.3 for nearly circular orbits ( $\mathrm{e}=0-0.25)$ and highly eccentric binaries $(e \sim 0.75)$, respectively. Although, previous surveys of stellar companions in a range of $\sim$ $3-50$ au have indicated that binary truncation might not be a primary mechanism for the clearing inner region of the disk (Pott et al. 2010; Kraus et al. 2012), there are different factors that prevented the detection of faint stellar companions in general, such as inner working angle and a small separation of the binary at the observing epoch.

In addition, a misleading interpretation of the SEDs can occur in the classification process of TDs through the SEDs of the CDs. Since an unresolved faint infrared companion can aggregate NIR flux to the net SED and if this object is surrounded by a disk, it could emit MIR levels similar to the MIR excess seen in the SED of TDs (e.g Duchêne et al. 2003; Kraus et al. 2015). Although, the SED of these CDs present several overlapping features with a "normal" SED of TDs, it would be misleading to treat them in a similar way. For instance, the implications for the presence of another star in the star-disk system entails an incorrect measurement of the luminosity and temperature, which translates into inaccurate age and mass estimates. This is the case of Coku Tau/4 and CS Cha that were originally described as TDs (Forrest et al. 2004; Espaillat et al. 2007), but eventually were presented as CDs (Ireland \& Kraus 2008; Guenther et al. 2007). This misclassification would be reflected in the estimation of birthplaces and timescales for formation of sub-stellar companions (brown dwarfs) and/or planetary systems, and the demographic properties of these populations (e.g. Najita, Andrews \& Muzerolle 2015).

Therefore, determining a more accurate relative picture of the lifetime of TDs and CDs requires a comprehensive survey capable of resolving close binaries $(\underset{\sim}{\sim} 30 \mathrm{au})$ and measuring their frequency in objects previously classified as TDs through their SEDs. Although, the open gap in the inner region of the disk might have different physical origins, in this paper we seek to identify if the dispersion of the primordial material in the inner region of the disk is a result of the tidal interaction between a close binary system and the disk. At small separations, detecting faint companions orbiting bright stars, that in addition, are surrounded by dusty material, can be challenging due to the high contrast between the companion and the primary star. However, observations of objects at early ages provide favorable IR contrast ratios for the detection of so far, unresolved faint companions because of their intrinsically higher luminosity $(\Delta \mathrm{K}<5 \mathrm{mag})$.

We use the Non-Redundant Mask interferometry (NRM) technique and NIRC2 instrument located at the Keck II telescope, which offers a solution to reach angular resolutions with the necessary contrast and is resistant to speckle noise in the image by measuring a self-calibrating quantity known as closure-phase (e.g. Martinache 2011). In order to achieve a higher accuracy in the detection limits of our data, the NRM completeness as a function of position and contrast utilizes a combination of a MonteCarlo Integration approach, giving a randomly sample of artificial binary stars, and Bayesian Inference, which uses prior probability density functions of the binary orbital parameters. We have restricted the selection of objects to regions with an age of $\sim 1-3$ Myrs and within a distance of about 220 pc. Taurus-Aurigae , IC348 region (Perseus) and Ophiucus star forming regions satisfy these criteria (Loinard et al. 2008; Wilking, Gagné \& Allen 2008).

This article is arranged as follows. In Section 2. we present the motivation for the sample selection, description of observations together with the data analysis and a review of the target properties such as distance to the star-forming regions and estimations of the inner radii. A simple Bayesian modelling analysis of these data is conducted in Section 3, with an emphasis on prior probabilities and description of binary and single models. The results of fitting to closure-phases in the $\chi^{2}$ minimization are synthesized with other information in the literature in Section 4. To perform a statistical Bayesian analysis of the fraction of the binarity as the main responsible mechanism opening the gaps in the TDs, we present a Jefreys Prior and its posterior probability in Section 5. Based on that analysis and observational results, we attempt to reconcile the observations with theoretical predictions from tidal interaction models and possible scenarios of planetary formation in Section 6. Finally, we provide an overall review of the work done and results in Section 7.

\section{TARGET SELECTION, OBSERVATIONS AND PROPERTIES}

In the past decade, the identified populations of $\mathrm{T}$ Tauri stars in the Taurus-Auriga, IC348 and Ophiuchus star forming regions have been well studied since the Spitzer data enriched the knowledge of dust distributions in the disks, providing large samples of Young Stellar Object members. Thus, we selected a sample in terms of their decreased flux (with respect to the CTTS median) in the wavelength range between $\sim 3$ and $24 \mu \mathrm{m}$, which tracks dust out to separations of at least $\sim 30 \mathrm{au}$. Our targets were selected based on clear inner regions in the disk seen in their SEDs. Sources with excess at wavelength in the range of $\sim 8$ to $24 \mu \mathrm{m}$ and a lack of excess between $\sim 3$ to $5 \mu \mathrm{m}$ are taken as disks with small or no dust excess in the inner region. Also, we included sources with strong emission between $\sim 3$ to $5 \mu \mathrm{m}$, but with a small excess emission at $\sim 8$ to $24 \mu \mathrm{m}$ and excess beyond $\sim 40 \mu \mathrm{m}$. The sample of objects were selected mostly from the work of Muzerolle et al. (2010); Cieza et al. (2010, 2012b); Espaillat et al. (2012) and Rebollido et al. (2015). These programs aimed to characterize SEDs for those objects in a "transition" phase and provided disk masses and accretion rates of the targets. The Two Micron All Sky Survey (2MASS) catalog Ks magnitudes are used to assign apparent magnitudes to these objects classified as TDs. To maximise sensitivity in our observations and achieve an image resolution of $\sim 20$ mas, we make use of the Kp filter to probe binary separations in this limit (e.g. Kraus et al. 2012). 

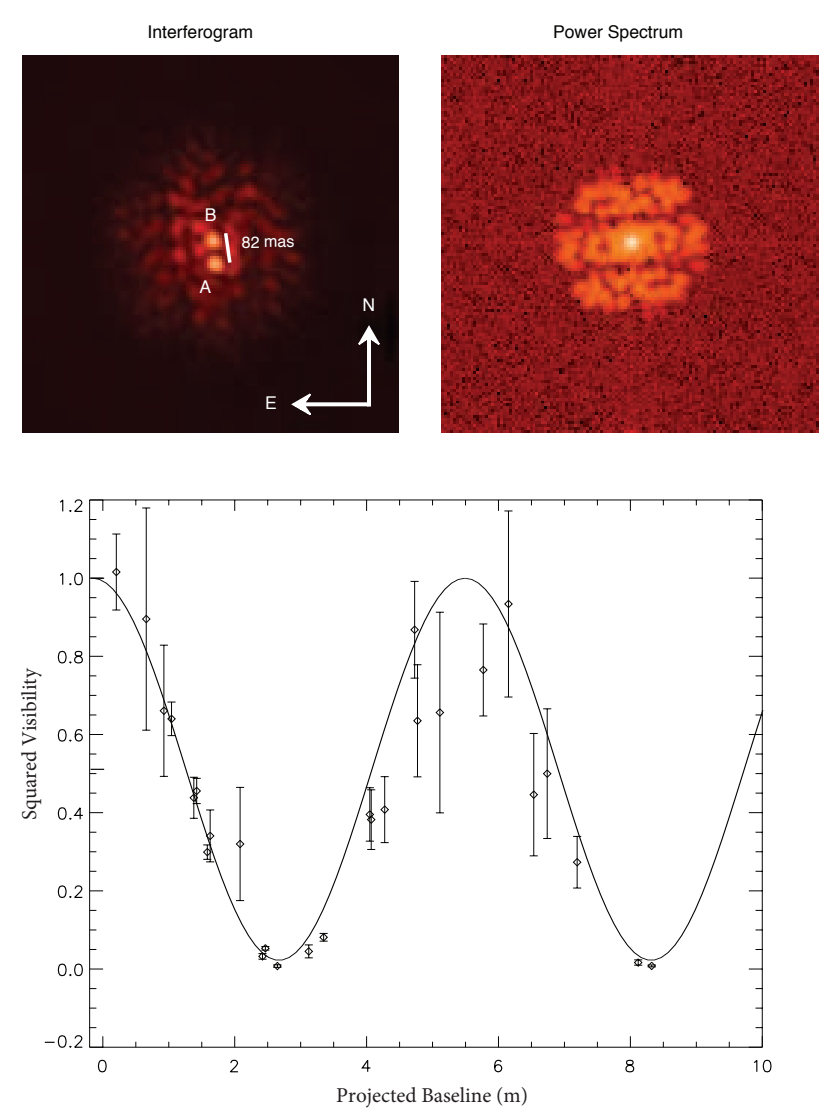

Figure 1. Top: Interferogram and Power spectrum of the new reported binary LRL 135. Bottom: Squared visibilities as a function of the projected baseline. The solid line shows the best-fit of the binary parameters, angular separation and position angle.

Considering that the maximum $\mathrm{Kp}-\mathrm{Ks}$ colour of our objects is only 0.10 mag 1 , we did not apply a magnitude conversion because it is less than the combination of our uncertainties in contrast ratio and the effects of stellar variability. Our final target list shown in Table 1 is composed of 24 transitional disks with $\mathrm{R}$ magnitudes brighter than 18 and spectral types in the range of G3-M5. For this final target list of TDs, only 2MASS J04210934+2750368 and EM* SR 24S have known stellar companions at 770 mas ( 108 au) (Cieza et al. 2010) and at 6000 mas ( 650 au) (Simon et al. 1995), respectively. These are not a close companion located in the range of our area of detection and do not affect the main purpose of the observations.

We observed our target list in August and December 2014 with the Adaptive Optics (AO) system of the near-infrared instrument (NIRC2) located at the Keck II 10 m telescope. The AO rotator tracking mode was set in vertical angle mode. A nine-hole mask located at the telescope pupil re-samples the light into a nonredundant interferogram of 36 pairwise fringes in the a Kp filter (Figure 1 top left panel). This pattern is specially designed to reach a near complete Fourier coverage. The Aladdin detector was configured to a 512 x 512 subarray and a multiple correlated double sampling readout mode was used in a narrow camera with a pixel scale of $9.952 \pm 0.002$ mas/pixel (Yelda et al. 2010). An overall exposure time of 20 seconds is used, except for the calibrators LRL 410, CIDA 2 and UX Tau A with 60, 5 and 5 seconds, respectively. Because some TDs have been previously observed using identical settings as our observational method, we access the Keck Observatory Archive (KOA) and complete our sample of TDs that were unfit to observe in our 2014 runs. Table 2 s shows a summary of the observational settings of the targets, calibrators and their observing epochs.

The observed data have been corrected by flat-fielding, removal of bad pixels and dark subtracted to be spatially filtered with a super-Gaussian function to maximise sensitivity Ireland et al. 2008). The aperture masking analysis is based on the extraction and calibration of closure-phase and squared-visibility, then carrying out least squares binary fitting. The interested reader can find a detailed description available in e.g. Kraus et al. (2016). In the case of fitting to binaries with an angular separation $(\rho)$ of $\gtrsim 25$ mas at high contrast, we fit only to closure-phase because of its immunity to changes in the AO point-spread function (PSF). We determined that any solutions with a significance of more than 6- $\sigma$ are detections of secondary components (Kraus et al. 2016). Then, we conducted a Bayesian analysis for marginal detections and contrast limits as described in section 3.1 As an input to this Bayesian analysis, for each set of calibrated closure phases, we computed a least squares fit to contrast (secondary/primary flux) in a grid of 80 x 80 models with 5 milli-arcsec spacing.

For reasons of both speed and in order to only consider the regime with symmetrical error bars, we approximated the contrast as being in the linear regime where closure phase is proportional to contrast. The output of this process was a grid of best fit contrasts and uncertainties. The uncertainties were scaled in order to acquire a $\chi^{2}$ equal to unity in the fit to the closure phases. For detections with $\rho<40$ mas and $\Delta \mathrm{K}<1 \mathrm{mag}$, we included visibility amplitudes for breaking contrast/separation degeneracies. When fitting to squared visibilities, we conservatively added a $20 \%$ miscalibration uncertainty in quadrature to the uncertainties estimated from scatter in our data, and also left, as a free parameter, the scaling of the interferometric visibilities. This was necessary because in AO data, Strehl ratios typically vary from target to PSF calibrator, and visibility amplitudes calibrate much more poorly than closure-phases. This miscalibration uncertainty needed to be added because Strehl variations, caused by e.g. changing atmospheric conditions, cause a variation in the visibility amplitudes between target and calibrator observations. Part of this uncertainty was taken into account by adding the scatter amongst calibrators in quadrature to the visibility amplitude uncertainty from the target.

Additionally, the detection limits are highly dependent on the contemporaneous observations of calibrators that must be single stars with high $\mathrm{S} / \mathrm{N}$ and ideally close to the observed target. The calibrators are used to remove effects of optical aberrations. Raw object visibilities are divided by calibrator visibilities, and calibrator closure phases are subtracted from raw object closure phases. However, we were not able to observe truly isolated stars in these dusty star-forming regions and for those observing epochs taken from KOA, we used likely single stars with non-redundant interferograms taken in the same observing run (Table 2). Therefore, in order to assure high $\mathrm{S} / \mathrm{N}$ and non-binarity in the set of objects to be used as calibrators in each epoch, we perform the following steps:

\footnotetext{
${ }^{1}$ We use the relation $\left(\mathrm{K}^{\prime}-\mathrm{K}\right) \cong 0.11(\mathrm{H}-\mathrm{K})$ from Vacca, Sheehy \& Graham (2007).
} 


\begin{tabular}{|c|c|c|c|c|c|c|c|c|c|c|c|}
\hline Target & Alter. Name & 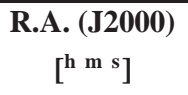 & $\begin{array}{c}\text { Dec }(\mathbf{J 2 0 0 0}) \\
{\left[{ }^{\circ}{ }^{\prime}{ }^{\prime \prime}\right]}\end{array}$ & $\begin{array}{c}\mathbf{R} \\
{[\mathrm{mag}]}\end{array}$ & $\begin{array}{c}\mathbf{J} \\
{[\mathrm{mag}]}\end{array}$ & $\begin{array}{c}\mathbf{H} \\
{[\mathrm{mag}]}\end{array}$ & $\begin{array}{c}\text { Ks } \\
{[\mathrm{mag}]}\end{array}$ & Sp. Type & $\begin{array}{c}\text { Log(Acc. Rate) } \\
{\left[\mathrm{M}_{\odot} \mathrm{yr}^{-1}\right]}\end{array}$ & $\begin{array}{c}\text { Inner Radii }^{\dagger} \\
\text { [au] }\end{array}$ & Reference $^{*}$ \\
\hline \multicolumn{12}{|c|}{ IC 348} \\
\hline $\begin{array}{l}\text { LRL } 21^{\S} \\
\text { LRL } 67 \\
\text { LRL } 72 \\
\text { LRL } 237 \\
\text { LRL } 97 \\
\text { LRL } 31 \\
\text { LRL 182 } \\
\text { LRL } 213 \\
\text { LRL 58 } \\
\text { LRL 135 } \\
\end{array}$ & $\begin{array}{l}\ldots \\
\cdots \\
\cdots \\
\cdots \\
\cdots \\
\cdots \\
\cdots \\
\cdots \\
\cdots \\
\cdots \\
\end{array}$ & $\begin{array}{l}034456.15 \\
034344.62 \\
034422.57 \\
034423.57 \\
034425.56 \\
034418.17 \\
034418.20 \\
034421.27 \\
034438.55 \\
034439.19 \\
\end{array}$ & 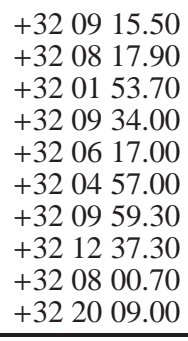 & $\begin{array}{l}14.81 \\
14.65 \\
15.93 \\
17.72 \\
18.32 \\
17.22 \\
18.10 \\
16.78 \\
16.43 \\
16.90 \\
\end{array}$ & $\begin{array}{l}11.02 \\
12.05 \\
12.12 \\
13.50 \\
12.98 \\
12.09 \\
13.22 \\
13.70 \\
11.94 \\
12.65 \\
\end{array}$ & $\begin{array}{c}9.99 \\
11.13 \\
11.15 \\
12.74 \\
11.70 \\
10.54 \\
12.27 \\
12.92 \\
10.90 \\
11.80 \\
\end{array}$ & $\begin{array}{c}9.47 \\
10.79 \\
10.79 \\
12.40 \\
11.14 \\
9.69 \\
11.87 \\
12.51 \\
10.47 \\
11.44 \\
\end{array}$ & $\begin{array}{c}\text { K0 } \\
\text { M0.75 } \\
\text { M2.5 } \\
\text { M5 } \\
\text { M2.25 } \\
\text { G6 } \\
\text { M4.25 } \\
\text { M4.75 } \\
\text { M1.25 } \\
\text { M4.5 } \\
\end{array}$ & $\begin{array}{c}-9.4 \\
-10.2 \\
<-11 \\
\mathrm{n} \\
\mathrm{n} \\
-7.9 \\
\mathrm{n} \\
\mathrm{n} \\
\mathrm{n} \\
\mathrm{y} \\
\end{array}$ & $\begin{array}{c}9 \\
10 \\
5 \\
0.005 \\
0.005 \\
14 \\
- \\
- \\
- \\
- \\
\end{array}$ & $\begin{array}{l}1,2,3,4,16 \\
1,2,3,4,16 \\
1,2,3,4,16 \\
1,2,3,5,15 \\
1,2,3,5,15 \\
1,2,3,4,6,16 \\
1,2,3,5 \\
1,2,3,5 \\
1,2,3,5 \\
1,2,3,5 \\
\end{array}$ \\
\hline \multicolumn{12}{|c|}{ Taurus-Aurigae } \\
\hline $\begin{array}{l}\text { IRAS04125+2902 } \\
\text { V410 X-ray } 6 \\
\text { J04210934+2750368 }\end{array}$ & $\begin{array}{c}\text { [GBA2007] } 527 \\
\ldots \\
\ldots\end{array}$ & $\begin{array}{lll}04 & 15 & 42.79 \\
04 & 19 & 01.11 \\
04 & 21 & 09.34 \\
\end{array}$ & $\begin{array}{l}+290959.77 \\
+281942.05 \\
+275036.84 \\
\end{array}$ & $\begin{array}{l}14.34 \\
16.50 \\
15.70 \\
\end{array}$ & $\begin{array}{l}10.71 \\
10.53 \\
11.23 \\
\end{array}$ & $\begin{array}{c}9.76 \\
9.60 \\
10.66 \\
\end{array}$ & $\begin{array}{c}9.38 \\
9.13 \\
10.36 \\
\end{array}$ & $\begin{array}{l}\text { M1.25 } \\
\text { M4.5 } \\
\text { M5.25 } \\
\end{array}$ & $\begin{array}{l}-9.5 \\
-10.85 \\
-10.3 \\
\end{array}$ & $\begin{array}{c}18-24 \\
- \\
- \\
\end{array}$ & $\begin{array}{l}1,2,7,8,12 \\
1,2,9,12 \\
1,2,9 \\
\end{array}$ \\
\hline \multicolumn{12}{|c|}{ Ophiuchus } \\
\hline $\begin{array}{l}\text { EM* SR 24S } \\
\text { EM* SR 21A } \\
\text { WSB 12 } \\
\text { J16262367-2443138 } \\
\text { J16273901-2358187 } \\
\text { WSB 63 } \\
\text { J16335560-2442049 } \\
\text { J16250692-2350502 } \\
\text { J16315473-2503238 } \\
\text { WSB 40 } \\
\text { V* V852 Oph } \\
\end{array}$ & $\begin{array}{c}\ldots \\
\ldots \\
\ldots \\
\text { DoAr } 25 \\
\text { DoAr } 33 \\
\ldots \\
\text { RX J1633.9-2442 } \\
\ldots \\
\text { WSB } 74 \\
\ldots \\
\ldots \\
\end{array}$ & $\begin{array}{lll}16 & 26 & 58.51 \\
16 & 27 & 10.28 \\
16 & 22 & 18.52 \\
16 & 26 & 23.68 \\
16 & 27 & 39.01 \\
16 & 28 & 54.07 \\
16 & 33 & 55.61 \\
16 & 25 & 06.91 \\
16 & 31 & 54.73 \\
16 & 26 & 48.65 \\
16 & 25 & 24.34 \\
\end{array}$ & $\begin{array}{llll}-24 & 45 & 36.87 \\
-24 & 19 & 12.74 \\
-23 & 21 & 48.10 \\
-24 & 43 & 13.86 \\
-23 & 58 & 18.70 \\
-24 & 47 & 44.20 \\
-24 & 42 & 05.00 \\
-23 & 50 & 50.30 \\
-25 & 03 & 23.80 \\
-23 & 56 & 34.20 \\
-24 & 29 & 44.30 \\
\end{array}$ & $\begin{array}{l}14.15 \\
13.50 \\
13.03 \\
12.99 \\
13.88 \\
15.41 \\
15.04 \\
15.55 \\
15.08 \\
15.42 \\
14.52 \\
\end{array}$ & $\begin{array}{c}9.75 \\
8.74 \\
9.52 \\
9.40 \\
9.90 \\
10.68 \\
10.46 \\
11.05 \\
10.14 \\
10.43 \\
10.75 \\
\end{array}$ & $\begin{array}{l}8.17 \\
7.51 \\
8.59 \\
8.40 \\
8.72 \\
9.43 \\
9.36 \\
9.97 \\
8.66 \\
9.18 \\
9.87 \\
\end{array}$ & $\begin{array}{l}7.06 \\
6.72 \\
8.11 \\
7.85 \\
8.21 \\
8.91 \\
8.80 \\
9.51 \\
7.75 \\
8.45 \\
9.45 \\
\end{array}$ & $\begin{array}{c}\text { K2 } \\
\text { G3 } \\
\text { K5 } \\
\text { K5 } \\
\text { K5.5 } \\
\text { M1.5 } \\
\text { K7 } \\
\text { M3 } \\
\text { K7 } \\
\text { K5.5 } \\
\text { M4.5 } \\
\end{array}$ & $\begin{array}{l}-8.0 \\
<-9.0 \\
-8.0 \\
-7.2 \\
-9.6 \\
-8.1 \\
-9.9 \\
-8.8 \\
-7.2 \\
- \\
- \\
\end{array}$ & $\begin{array}{c}29 \\
25 \\
- \\
- \\
- \\
1.9 \pm 0.3 \\
7.9 \pm 2.3 \\
4.8 \pm 2.5 \\
- \\
- \\
- \\
\end{array}$ & $\begin{array}{l}1,17 \\
1,2,13 \\
1,2,10 \\
1,2,10 \\
1,2,10 \\
1,2,10,14 \\
1,2,10,14 \\
1,10,14 \\
1,10 \\
1,2,11 \\
1,2,11 \\
\end{array}$ \\
\hline
\end{tabular}

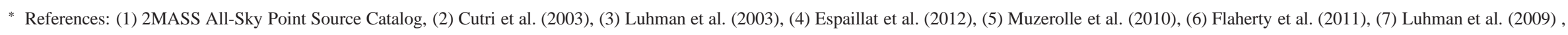

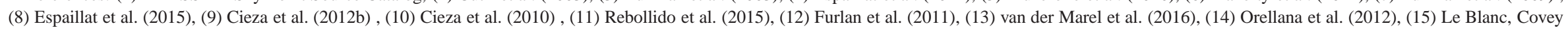
\& Stassun (2011), (16) Espaillat et al. (2010), (17) Andrews et al. (2011)

$\dagger$ Inner radii from literature.

* Stellar parameters taken from Andrews et al. (2011).

$\S$ Target IDs of IC348 members are taken from the acronym Cl* IC 348 from Luhman et al. (1998).

II Targets with 2MASS identifiers are presented by their designation e.g. J04210934+2750368 
Table 2: Summary of Observations.

\begin{tabular}{|c|c|c|c|c|c|c|c|}
\hline ID & $\begin{array}{c}\text { BJD } \\
(2400000+)\end{array}$ & $\begin{array}{l}\mathbf{t}_{i n t} \\
{[\mathbf{s}]}\end{array}$ & Coadds & $\mathbf{N}_{\text {frames }}$ & Airmass & Type & Note \\
\hline \multicolumn{8}{|c|}{ June 18, 2008} \\
\hline Haro 1-6 & 54635.75 & 5.00 & 4 & 7 & 1.92 & Calibrator & $>5 \sigma$ \\
\hline RX J1620.9-2352 & 54635.75 & 5.00 & 4 & 7 & 1.82 & Calibrator & \\
\hline$E M * S R 24 S$ & 54635.75 & 5.00 & 4 & 6 & 1.86 & Target & \\
\hline $\mathrm{EM}^{*} \mathrm{SR} 24 \mathrm{~S}$ & 54635.75 & 5.00 & 4 & 8 & 1.81 & Target & \\
\hline Haro $1-6$ & 54635.75 & 5.00 & 4 & 7 & 1.72 & Calibrator & \\
\hline Haro 1-6 & 54635.79 & 1.00 & 10 & 11 & 1.53 & Calibrator & \\
\hline EM* SR 24S & 54635.79 & 1.00 & 10 & 7 & 1.51 & Target & \\
\hline EM* SR 21A & 54635.88 & 1.00 & 20 & 7 & 1.40 & Target & \\
\hline $\mathrm{EM}^{*} \mathrm{SR} 24 \mathrm{~N}$ & 54635.88 & 2.50 & 8 & 2 & 1.41 & Calibrator & $>5 \sigma$ \\
\hline J16262367-2443138 & 54635.88 & 2.50 & 8 & 7 & 1.42 & Target & \\
\hline WSB 12 & 54635.88 & 2.50 & 8 & 7 & 1.40 & Target & \\
\hline EM* SR 21A & 54635.88 & 2.50 & 8 & 7 & 1.43 & Target & \\
\hline $\mathrm{J} 16262367-2443138$ & 54635.88 & 2.50 & 8 & 7 & 1.45 & Target & \\
\hline WSB 12 & 54635.88 & 2.50 & 8 & 7 & 1.43 & Target & \\
\hline EM* SR 21A & 54635.92 & 2.50 & 8 & 7 & 1.47 & Target & \\
\hline J16262367-2443138 & 54635.92 & 2.50 & 8 & 7 & 1.50 & Target & \\
\hline WSB 12 & 54635.92 & 2.50 & 8 & 7 & 1.49 & Target & \\
\hline V* V2059 Oph & 54635.92 & 20.00 & 1 & 7 & 1.52 & Calibrator & $>5 \sigma$ \\
\hline RX J1625.2-2455 & 54635.92 & 20.00 & 1 & 7 & 1.58 & Calibrator & \\
\hline $\mathrm{V}^{*} \mathrm{~V} 2059 \mathrm{Oph}$ & 54635.92 & 20.00 & 1 & 7 & 1.61 & Calibrator & \\
\hline
\end{tabular}

November 03, 2009

\begin{tabular}{lcccccc}
\hline J04183030+2743208 & 55138.92 & 10.00 & 1 & 10 & 1.05 & calibrator \\
J04380083+2558572 & 55138.96 & 10.00 & 1 & 10 & 1.01 & calibrator \\
J04350850+2311398 & 55139.00 & 20.00 & 1 & 10 & 1.01 & calibrator \\
V410 X-ray 6 & 55139.00 & 10.00 & 1 & 9 & 1.03 & Target \\
J04244506+2701447 & 55139.04 & 10.00 & 1 & 9 & 1.05 & calibrator \\
\hline
\end{tabular}

April 23, 2011

\begin{tabular}{lccccccc}
\hline J16233462-2308467 & 55674.92 & 5.00 & 4 & 12 & 1.67 & Calibrator & $>5 \sigma$ \\
WSB 63 & 55674.92 & 5.00 & 4 & 10 & 1.65 & Target & \\
J16273901-2358187 & 55674.96 & 5.00 & 4 & 9 & 1.55 & Target & \\
J16250692-2350502 & 55674.96 & 5.00 & 4 & 10 & 1.49 & Target & $>4 \sigma$ \\
DoAr 32 & 55674.96 & 5.00 & 4 & 11 & 1.47 & Calibrator & $>47$ \\
WSB 63 & 55674.96 & 5.00 & 4 & 10 & 1.46 & Target & $>5 \sigma$ \\
BKLT J162624-244323 & 55674.96 & 5.00 & 4 & 11 & 1.44 & Calibrator & $>50$ \\
WSB12 (RX J1622.3-2321) & 55675.00 & 5.00 & 4 & 11 & 1.38 & Target & \\
J16315473-2503238 & 55675.00 & 5.00 & 4 & 10 & 1.42 & Target & \\
BKLT J162624-244323 & 55675.00 & 5.00 & 4 & 10 & 1.40 & Calibrator & \\
EM* SR 8 & 55675.00 & 5.00 & 4 & 10 & 1.40 & Calibrator & Target \\
J16335560-2442049 & 55675.00 & 5.00 & 4 & 11 & 1.40 & Calibrator & \\
DoAr 50 & 55675.00 & 5.00 & 4 & 10 & 1.42 & Carget & \\
WSB 63 & 55675.04 & 5.00 & 4 & 10 & 1.41 & Target & \\
J16335560-2442049 & 55675.04 & 5.00 & 4 & 10 & 1.41 & Target & \\
DoAr 50 & 55675.04 & 5.00 & 4 & 9 & 1.44 & Calibrator & $>5 \sigma$ \\
DoAr 24 & 55675.04 & 5.00 & 4 & 10 & 1.44 & Calibrator & \\
2E 1624.2-2444 & 55675.04 & 5.00 & 4 & 11 & 1.49 & Calibrator & \\
RX J1633.9-2442 & 55675.08 & 5.00 & 4 & 10 & 1.48 & Target & \\
RX J1624.8-2359 & 55675.08 & 5.00 & 4 & 11 & 1.51 & Calibrator & no-Binary \\
ROXs 4 & 55675.08 & 5.00 & 4 & 11 & 1.57 & Calibrator & \\
2E 1624.6-2352 & 55675.08 & 5.00 & 4 & 11 & 1.60 & Calibrator & \\
IRAS 16114-1858 & 55675.08 & 5.00 & 4 & 9 & 1.62 & Calibrator & \\
\hline
\end{tabular}

November 15, 2011

\begin{tabular}{|c|c|c|c|c|c|c|c|}
\hline MBO 22 & 55880.75 & 5.00 & 4 & 25 & 1.50 & Calibrator & $>5 \sigma$ \\
\hline LRL 21 & 55880.79 & 5.00 & 4 & 23 & 1.42 & Target & \\
\hline LRL 72 & 55880.79 & 5.00 & 4 & 23 & 1.33 & Target & \\
\hline MBO 22 & 55880.79 & 5.00 & 4 & 23 & 1.22 & Calibrator & \\
\hline LRL 21 & 55880.83 & 5.00 & 4 & 23 & 1.22 & Target & \\
\hline LRL 67 & 55880.83 & 5.00 & 4 & 21 & 1.18 & Target & \\
\hline LRL 21 & 55880.83 & 5.00 & 4 & 22 & 1.11 & Target & \\
\hline
\end{tabular}


Table 2 -Continued

\begin{tabular}{|c|c|c|c|c|c|c|c|}
\hline ID & $\begin{array}{c}\text { BJD } \\
(2400000+)\end{array}$ & $\begin{array}{l}\mathbf{t}_{i n t} \\
{[\mathbf{s}]}\end{array}$ & Coadds & $\mathbf{N}_{\text {frames }}$ & Airmass & Type & Note \\
\hline MBO 22 & 55880.83 & 5.00 & $\overline{4}$ & $\overline{2}$ & 1.07 & Calibrator & \\
\hline LRL 67 & 55880.88 & 5.00 & 4 & 23 & 1.06 & Target & \\
\hline $\mathrm{J} 03302409+3114043$ & 55880.88 & 5.00 & 4 & 23 & 1.03 & Calibrator & $>5 \sigma$ \\
\hline V410 X-ray 6 & 55880.88 & 5.00 & 4 & 23 & 1.05 & Target & \\
\hline $\mathrm{J} 04300424+3522238$ & 55880.92 & 5.00 & 4 & 18 & 1.07 & Calibrator & $>5 \sigma$ \\
\hline V410 X-ray 6 & 55880.92 & 5.00 & 4 & 21 & 1.02 & Target & \\
\hline $\mathrm{J} 04300424+3522238$ & 55880.92 & 5.00 & 4 & 19 & 1.04 & Calibrator & \\
\hline HBC 390 & 55880.96 & 5.00 & 4 & 22 & 1.04 & Calibrator & \\
\hline $\mathrm{J} 04303235+3536133$ & 55880.96 & 5.00 & 4 & 10 & 1.04 & Calibrator & no-Binary \\
\hline $\mathrm{J} 04303235+3536133$ & 55880.96 & 5.00 & 4 & 11 & 1.04 & Calibrator & no-Binary \\
\hline $\mathrm{J} 03302409+3114043$ & 55880.96 & 5.00 & 4 & 14 & 1.09 & Calibrator & $>5 \sigma$ \\
\hline
\end{tabular}

April 14, 2012

\begin{tabular}{llllllll}
\hline & & & & & \\
RX J1615.3-3255 & 56032.04 & 5.00 & 4 & 12 & 1.66 & Calibrator & \\
RX J1615.9-3241 & 56032.04 & 5.00 & 4 & 13 & 1.66 & Calibrator & \\
RX J1625.3-2402 & 56032.08 & 5.00 & 4 & 3 & 1.42 & Calibrator & no-HighBF \\
V* V852 Oph & 56032.08 & 5.00 & 4 & 3 & 1.45 & Target & \\
WMR2005 1-38 & 56032.08 & 5.00 & 4 & 3 & 1.47 & Calibrator & \\
WSB 40 & 56032.08 & 5.00 & 4 & 3 & 1.50 & Target \\
WMR2005 1-21 & 56032.12 & 5.00 & 4 & 3 & 1.61 & Calibrator & \\
\hline
\end{tabular}

August 11, 2014

\begin{tabular}{lccccccc}
\hline V* V711 Per & 56881.04 & 20.00 & 1 & 8 & 1.38 & Calibrator & $>5 \sigma$ \\
LRL 110 & 56881.04 & 20.00 & 1 & 6 & 1.35 & Calibrator & \\
LRL 410 & 56881.04 & 60.00 & 1 & 5 & 1.31 & Calibrator & \\
CIDA 2 & 56881.08 & 5.00 & 4 & 8 & 1.36 & Calibrator & $>5 \sigma$ \\
IRAS 04125+2902 & 56881.12 & 20.00 & 1 & 7 & 1.16 & Target & \\
V410 X-ray 3 & 56881.12 & 20.00 & 1 & 5 & 1.13 & Calibrator & $>5 \sigma$ \\
UX Tau A & 56881.12 & 5.00 & 4 & 8 & 1.14 & Calibrator & \\
\hline
\end{tabular}

August 12, 2014

\begin{tabular}{lllllll}
\hline LRL 75 & 56882.04 & 20.00 & 1 & 8 & 1.54 & Calibrator \\
LRL 40 & 56882.04 & 20.00 & 1 & 8 & 1.46 & Calibrator \\
LRL 168 & 56882.04 & 20.00 & 1 & 8 & 1.42 & Calibrator \\
LRL 97 & 56882.04 & 20.00 & 1 & 8 & 1.35 & Target \\
LRL 237 & 56882.04 & 20.00 & 1 & 6 & 1.31 & Target \\
\hline
\end{tabular}

August 13, 2014

\begin{tabular}{llllllll}
\hline KOI-137 & 56883.00 & 20.00 & 1 & 7 & 1.65 & Calibrator \\
KOI-0044 & 56883.00 & 20.00 & 1 & 6 & 1.66 & Calibrator \\
KOI-4567 & 56883.00 & 20.00 & 1 & 7 & 1.66 & Calibrator \\
LRL 72 & 56883.04 & 20.00 & 1 & 7 & 1.55 & Target & \\
J04311907+2335047 & 56883.08 & 20.00 & 1 & 7 & 1.37 & Calibrator & $>5 \sigma$ \\
\hline
\end{tabular}

December 09, 2014

\begin{tabular}{llllllll}
\hline LRL 53 & 57000.75 & 20.00 & 1 & 7 & 1.19 & Calibrator \\
LRL 182 & 57000.75 & 20.00 & 1 & 8 & 1.17 & Target \\
LRL 58 & 57000.79 & 20.00 & 1 & 8 & 1.13 & Target \\
LRL 355 & 57000.79 & 20.00 & 1 & 7 & 1.12 & Calibrator & \\
LRL 135 & 57000.79 & 20.00 & 1 & 7 & 1.10 & Target & $>5 \sigma$ \\
LRL 233 & 57000.79 & 20.00 & 1 & 7 & 1.08 & Calibrator & $>50$ \\
LRL 31 & 57000.79 & 20.00 & 1 & 4 & 1.07 & Target & \\
LRL 169 & 57000.79 & 20.00 & 1 & 7 & 1.06 & Calibrator & \\
LRL 213 & 57000.83 & 20.00 & 1 & 7 & 1.05 & Target & \\
J03302409+3114043 & 57000.96 & 20.00 & 1 & 13 & 1.41 & Calibrator & $>5 \sigma$ \\
MBO 22 & 57001.00 & 20.00 & 1 & 7 & 1.46 & Calibrator & $>5 \sigma$ \\
J04210934+2750368 & 57001.00 & 20.00 & 1 & 3 & 1.25 & Target & \\
J04300424+3522238 & 57001.00 & 20.00 & 1 & 8 & 1.30 & Calibrator & \\
J04303235+3536133 & 57001.00 & 20.00 & 1 & 6 & 1.33 & Calibrator & no-binary \\
Pr 0211 & 57001.08 & 20.00 & 1 & 7 & 1.02 & Calibrator & \\
Pr 0225 & 57001.08 & 20.00 & 1 & 7 & 1.02 & Calibrator & \\
\hline \hline
\end{tabular}


(i) We first identified the set of targets of obvious binarity, e.g LRL 135 shown in Figure 1 and targets with significance levels of $>8-\sigma$ by fitting only to closure-phase and then, removed them from the source sample of calibrators.

(ii) The remaining objects, calibrators and science targets, play the role of inter-calibrating sources. After fitting closure phases for every observed object, we started eliminating from the set of calibrator sources those objects with a significance of more than $5-\sigma$, assuring an isolated object with high $\mathrm{S} / \mathrm{N}$.

(iii) The closure phases of those remaining objects are used as the final set of calibrators.

In our survey, the aperture masking data identified a well resolved a nearly equal luminosity companion for LRL 135 at 82 mas with a $\Delta \mathrm{K}$ of $0.17 \pm 0.01$ and a position angle of $208^{\circ}$, which is shown in Figure 1 as the observed interferogram and its power spectrum. We provide a more detailed description about these results in Section 4.1, including reference to maser distances. Also, the extracted squared visibilities are plotted, where clearly fringe contrast goes to near zero at the longest baseline of the mask $(\sim 9$ $\mathrm{m})$.

\subsection{Target Properties}

Our methodology to identify companions that might be responsible for the observed TD SEDs and could be orbiting in the inner region of the disk, requires estimations of the distances to the inner disk wall from the central star $\left(r_{d}\right)$. However, not all TDs have a previous measurements of the inner radii and are not calculated by following a standard approach. Because we seek for uniformity in these estimations, we developed a simple approach highly dependent on the stellar luminosities $\left(L_{\star}\right)$ and the dust temperature in the disk $\left(T_{d}\right)$. The stellar luminosities are calculated with the dereddened $J$-band photometry method from Kenyon \& Hartmann (1995) and adopting the known distances to each different star-forming region. We de-reddened the $J$-band fluxes using the $\mathrm{A}_{J}$ extinction and measured by following the Mathis (1990) extinction law with $A(\lambda) / A(J) \sim(\lambda / 1.25)^{-\alpha}$, where $\alpha=1.7$. We used $A_{J}=2.62\left[(J-H)-(J-H)_{0}\right]$, where $(J-H)_{0}$ is the expected colour of a main-sequence star from Pecaut \& Mamajek (2013). We derived the stellar properties based on the spectral types taken from literature and a conversion to the effective temperatures $\left(\mathrm{T}_{\mathrm{eff}}\right)$ taken from Pecaut \& Mamajek (2013) with uncertainties of $\sim 150$ $\mathrm{K}$, corresponding to \pm 1 spectral subclass (Table 1 . Additionally, using $\mathrm{T}_{\text {eff }}$ and $\mathrm{L}_{\star}$, and assuming a single star system, we estimated the stellar masses $\left(M_{\star}\right)$ for each TD. Those in the range between 0.01 and 1.4 $M_{\odot}$, were derived from Baraffe et al. (2015) and stellar masses $>1.4 M_{\odot}$ from the PARSEC evolutionary models (Bressan et al. 2012). For the unknown metallicity values we adopted solar composition, and we also held the surface gravity fixed at the value $\log g=4.0$, typical for PMS stars. Table 1 shows $\mathrm{R}$ and Ks magnitudes, spectral types and stellar properties of these objects.

Distances to the star forming regions were adopted from literature. Thus, a distance of $140 \pm 10 \mathrm{pc}$ was adopted to the TaurusAurigae star forming region (Loinard et al. 2008). However, we found a large range of discrepancies in the distances to Ophiuchus and IC-348 members, leading us to carefully choose the most appropriate values, since these young members placed in the $\mathrm{H}-\mathrm{R}$ diagram are sensitive to any variation of these distances (Figure 2]. We adopted a distance to the IC 348 Region based on the distance to the Perseus molecular cloud, that has been estimated in a wide range between 220 - 380 pc (Harris, Morgan \& Roman 1954;
Herbig \& Jones 1983; Cernis 1993; Scholz et al. 1999; de Zeeuw et al. 1999; Hirota et al. 2008; Hirota 2010). We examined in detail the distance to the brightest cluster member, LRL 1. As a pair of B5V stars, these objects are zero-age main sequence (ZAMS) so they have a model-derived luminosity that is almost independent of age. Using the same technique as described above, we confirmed the distance of $220 \pm 10 \mathrm{pc}$, excluding ZAMS model uncertainties but including uncertainties in reddening. We provide a more detailed discussion about the IC 348 distance in Appendix A. In the case of the distance to the Ophiuchus region and as the position of most of our objects sit around the main cloud, L1688, we based our decision on the distance measured to this association. For our purposes and due to the wide extension in the position of the TDs, we adopted a mean distance of 130 pc to L1688 core (Wilking, Gagné \& Allen 2008, and references therein) and consistent with the distance of $131 \pm 3$ pc calculated by Mamajek (2008).

Once the distances were found and constrained, we calculated the bolometric luminosities $\left(L_{\star}\right)$ of IC 348, Ophiuchus and TaurusAuriga members. Estimations of stellar masses and ages are obtained from the H-R diagram and Baraffe et al. (2015) evolutionary tracks, except for IC348-21, IC348-31 and EM* SR 21A, where we made use of the PARSEC evolutionary models (Bressan et al. 2012). These values are estimated with the use of $L_{\star}$ and $K$ absolute magnitudes. Age and stellar mass uncertainties are based mainly on the H-R diagram placement and the determination of $\mathrm{L}_{\star}$, since $\mathrm{T}_{\text {eff }}$ does not vary with a large magnitude at ages $<5$ Myrs in the pre-main sequence evolution tracks of low mass stars. The main sources of error on the $\mathrm{L}_{\star}$ uncertainties are distance and extinction (Hartmann 2001). In our estimates of $A_{J}$, we used the expected colour of a main-sequence star, which underestimates these values up to a factor of 2, and it is reflected in the luminosity and age of the system. In the special case of embedded object EM* SR 24S that belongs to the triple system EM* SR24, we adopted stellar properties from Andrews et al. (2011) to avoid any IR contribution from its nearby binary companion, EM* SR 24N (0".2; Simon et al.1995). Table 3 shows the estimated stellar parameters. Figure 2 shows Baraffe evolutionary models with IC348, Taurus-Aurigae and Ophiuchus members. They are dispersed in a range of ages between 0.5 and 10 Myrs, characteristic of T-Tauri stars. Here, we carefully selected the chosen isochrone to derive stellar masses for each target, because these isochrones are also used in our Bayesian analysis, as explained in section 3.1

\subsubsection{Dust Temperature and Inner Radii}

As demonstrated by Espaillat et al. (2012), the NIR excesses of classical TDs are well reproduced by the emission of a vertical wall directly exposed to stellar radiation. Then, we have computed the TD SEDs from NIR to MIR wavelengths in order to estimate inner wall radii for every disk (Figure 3). Since the thermal balance between emission and absorption of radiation is dominated by the dust grains as the main opacity source, we computed the dust temperature $\left(T_{d}\right)$ at the truncation radius $\left(r_{d}\right)$ of the disk. Our input photometry for SED fitting were from 2MASS (1.25, 1.65, 2.22 $\mu \mathrm{m})$ and Spitzer/IRAC (3.6, 4.5, 5.8, 8 and $24 \mu \mathrm{m})$ (Skrutskie et al. 2006; Evans et al. 2003, 2009; Currie \& Kenyon 2009; Rebull et al. 2010).

An estimation of the $T_{d}$ was computed by fitting the stellar photosphere+disk black body function $\left(F_{m}\right)$ to the observed data $\left(F_{v}\right)$ and minimizing the sum of squares. Prior to this fitting, the photometric data were dereddened using the Mathis (1990) approach, since the properties of the inner disk material significantly 
Table 3. Luminosity and Extinction for Our Sample Members.

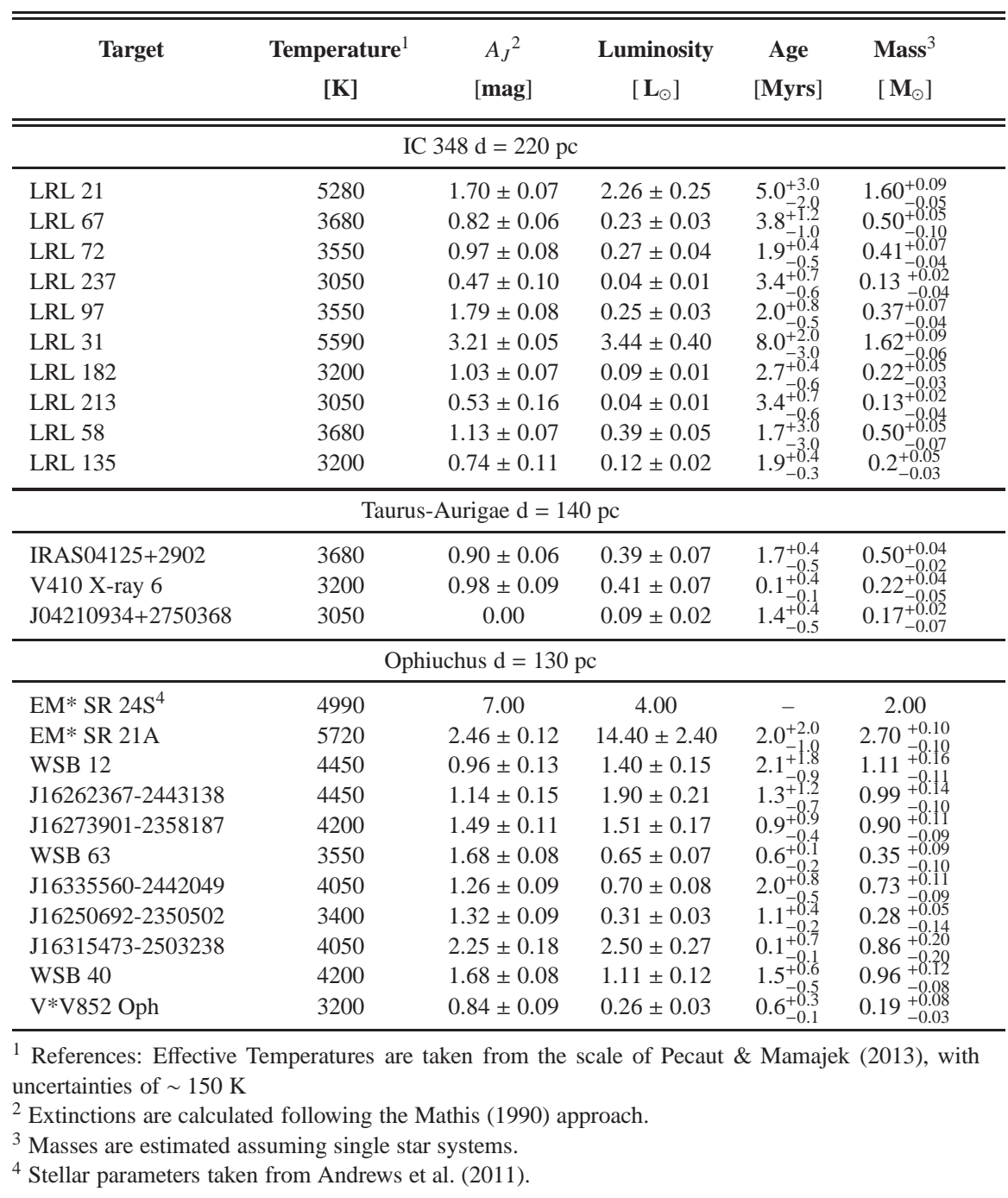

affects flux emission in these bands, and thus, the inner radii approximations (Furlan et al. 2011). To calculate the stellar synthetic photometry with a fixed temperature $T_{\star}$, which is approximated by the $\mathrm{T}_{\text {eff }}$, we 1) interpolated the response curves, for the set of filters used in the fitting, and the BT-Settl spectra models of the corresponding $T_{\star}$ Allard 2014) and 2) convolved the filter response curves with the syntethic spectra, to match the spectral resolution. Because the 2MASS, IRAC, and $24 \mu \mathrm{m}$ data have a photometric uncertainty of between a few percent and 0.1 mag for the objects investigated here, systematic effects can contribute up to $0.1 \mathrm{mag}$ and also, to account for flux variability of the objects, we added an observational error of $12 \%$. A multiplicative dilution factor relating the central star radius $\left(R_{\star}\right)$ and the distance to the object $(d)$ is part of the minimization of the $\chi^{2}$. Then, the model of received flux is the product of a dilution factor and the blackbody flux. In the case of a star, this dilution factor is given by $M_{d}=\left(\frac{R_{\star}}{d}\right)^{2}$ (e.g. Bayo et al. 2008)

Finally, the estimations of the truncation radius, $r_{d}$, are obtained by assuming Local Thermodynamic Equilibrium, and the ability of the dust to acquire thermal balance between absorption and emission of the radiation. The inner wall, nearly perpendicular to the stellar radiation, is heated only by the central star with a characteristic $R_{\star}$ and $T_{\star}$. Additionally, if the scattering of the dust grains is negligible and assuming optically thin gas in the inner region, we have in radiative equilibrium that the inner wall is being truncated at:

$$
r_{d}=\frac{R_{\star}}{2 \sqrt{ } \varepsilon}\left(\frac{T_{\star}}{T_{d}}\right)^{2}
$$

where $\varepsilon \equiv \frac{\kappa\left(T_{d}\right)}{\kappa\left(T_{\star}\right)}$ is the thermal cooling efficiency factor that characterizes the dust properties of a certain size (Dullemond \& Monnier 2010). If the inner wall consist of small dust grains of radius $a<<3 \mu \mathrm{m}$ and the backwarming by the grains deeper in the wall is negligible, then $\varepsilon<<3^{-0.5}$. For our purposes, the size of the grains at the location of the inner wall is taken to be $a \sim 0.1 \mu \mathrm{m}$, leading to estimations of $r_{d}$ with $\varepsilon \sim 0.08$ (Isella \& Natta 2005, and references therein) as shown in Table 6 For EM* SR 24S, because of its large inclination and elongated ring with a significant brightness asymmetry along the major axis, and to avoid any flux contamination in the Spitzer/IRAC bands from EM* SR 24N, we adopted the inner radius estimated by Andrews et al. (2011), and recently confirmed by van der Marel et al. (2015). Our $r_{d}$ estimations are in agreement with those previously measured (Table 1 ). 


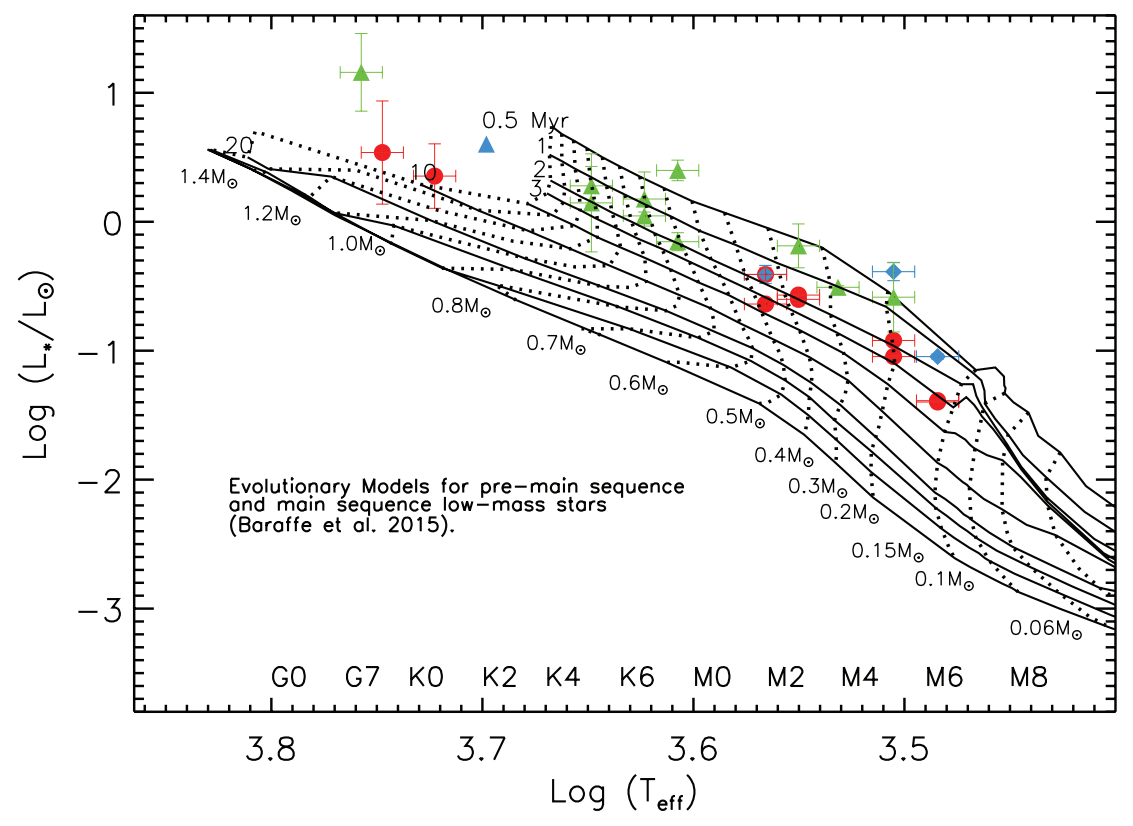

Figure 2. Theoretical models from Baraffe et al. (2015) for low mass young stars. Solid lines in descending order are 0.5, 1, 2, 3, 5, 10, 20, 50 and 100 Myrs isochrones and dashed lines represent the evolutionary tracks in the range of 0.06 and $1.4 \mathrm{M}$. Blue solid diamonds, green solid triangles and red solid dots are TDs from Taurus-Aurigae, Ophiuchus and IC348 star forming regions, respectively. The blue solid triangle corresponds to EM* SR $24 \mathrm{~S}$ with $L_{\odot}$ and T (K) taken from Andrews et al. (2011). We used the scale temperatures range from Pecaut \& Mamaiek (2013) and stellar luminosities are estimate as described in section 2.1

\section{DATA ANALYSIS}

\subsection{Bayesian Analysis}

Although some secondary companions are observed in the interferograms, e.g. Figure 1 it is important to carefully account for the assumptions inherent in the imaging completeness. A conservative approach where only secure detections are considered and conservative detection limits are quoted does not make maximum use of the data, especially at the smallest separations where binaries of moderate contrast ratios give relatively small closure-phase signals. Here, we made use of Bayesian statistics to compute confidence levels for detections, providing the advantage of using prior information of the underlying population of faint stellar companions. In essence, our approach to completeness correction, along with extensive Monte Carlo simulations, assigns the probability of detecting the presence of a faint companion or absence of it. We built two hypotheses, binary $\left(B_{n}\right)$ and single $\left(S_{n}\right)$, by using prior information of these models together with the aperture masking data of the TDs. Thus, a Bayes' theorem expresses the strengths of the hypotheses as follows:

$$
\begin{aligned}
& p(B \mid D)=\frac{p\left(B_{n}\right) p\left(D \mid B_{n}\right)}{p(D)} \\
& p(S \mid D)=\frac{p\left(S_{n}\right) p\left(D \mid S_{n}\right)}{p(D)}
\end{aligned}
$$

where $p\left(D \mid B_{n}\right)$ and $p\left(D \mid S_{n}\right)$ are the global likelihood func-

\footnotetext{
2 The global likelihood of a model is equal to the weighted average likelihood for all the parameters in consideration.
}

tions or probability of obtaining data $\mathrm{D}$, if $B_{n}$ or $S_{n}$ are true; $p\left(B_{n}\right)$ and $p\left(S_{n}\right)$ are the prior probabilities; and $p\left(B_{n} \mid D\right)$ and $p\left(S_{n} \mid D\right)$ are the posterior probabilities of $B_{n}$ and $S_{n}$, respectively. The index $n$ represents the number of simulations performed to determine the probability for each model.

\subsubsection{Global Likelihood: Confirming or Ruling out the Presence of a Binary System.}

In the data reduction process, after computing closure phases, the calibrated data set is used to search for faint companions close to the central star and orbiting the inner region of the disk. Our search strategy is based on the computation of the global likelihood that can be maximized from the conditional likelihood and its joint prior probability (Equation 4). Here, the conditional likelihood expresses the probability of observing our data for a specific set of model parameters and is weighted by the joint prior probability that incorporates prior information about the distribution of the model parameters. In our case, we have two hypotheses, $B_{n}$ and $S_{n}$, that can be tested by computing their global likelihood as follows:

3.1.1.1 Confirming a Binary System: The maximum global likelihood or the odds by which our data favors a binary model, lies in our approach to completeness correction and the extensive binary star simulations to assign all possible contrasts of the secondary relative to the primary $(C)$, angular separation $(\rho)$ and position angle $(\theta)$ values, as detailed below. Thus, our analysis to compute confidence levels is based on the derivation of the $\chi^{2}$ goodness-of-fit to the $n$ mock binary system models. From Equation 2 and marginalizing over all possible model parameters, we have that the global likelihood for a binary system model is: 


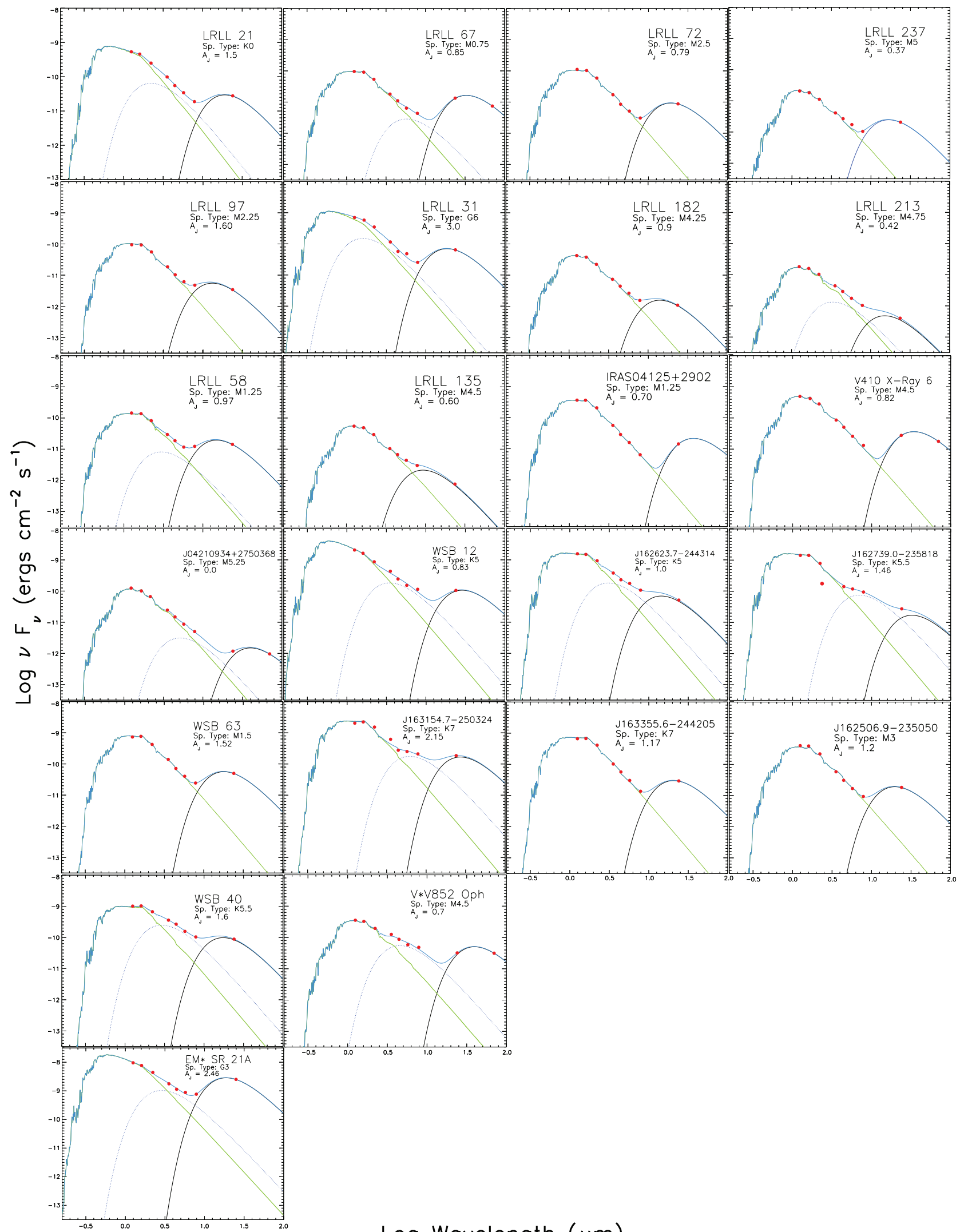

Log Wavelength $(\mu \mathrm{m})$

Figure 3. Spectral energy distribution of the sources classified as transitional disks. Red dots show photometric data acquired from the literature, green line is the BT-settl spectrum model according to the spectral type, blue line is the best fit, and the solid black and dotted blue lines are the disk black-body function values. $A_{J}$ values used are in Table 3 


$$
P\left(D \mid B_{n}\right)=\int d \Psi p\left(\Psi \mid B_{n}\right) \times P\left(D \mid B_{n}, \Psi\right)
$$

where, $P\left(D \mid B_{n}, \Psi\right)$ is the conditional likelihood, $P\left(\Psi \mid B_{n}\right)$ is the joint prior probability for the model parameters and $\Psi=$ $(T, a, e, \Omega, \omega, i, t, q)$ is the eight-dimensional parameter space representing all the possible binary orbits with the orbital parameters: Time of periastron passage ( $T$, years), Semi-major axis ( $a$, arcsecond), Eccentricity $(e)$, Position angle of the line of nodes $(\Omega)$, Longitude of periatron $(\omega)$, Inclination ( $i$, degrees), Epoch of observation $(t$, year) and Mass ratio $(q)$.

To compute the eight-dimensional integral shown in Equation 4 we made use of Monte Carlo Integration by generating a number of random samples according to a determined probability distribution function (PDF) in a specific spatial domain. The prior PDFs for $T, \Omega$ and $\omega$ for a binary system are assumed to have a uniform distribution (uninformative prior), so $p\left(T, \Omega, \omega_{2} \mid B_{i}\right)=1$ in the space domain of these parameters. The inclination $i$ is sampled considering that the orbital plane of the generated binaries might have any orientation in space. For $q$, we considered the mass ratio distribution often modelled in the form $f(q) \propto q^{\beta}$, where $\beta$ is a power index of 0 that reasonably describes our TD sample with spectral types ranging G3-M5 (e.g. Janson et al. 2012; Raghavan et al. 2010). For the prior distribution of $e$ for these very young objets in a process of orbital circularisation, we based our approach on our resolving limitations of up to $\gtrsim 2$ au that corresponds to orbital periods of $\sim 1000$ days and is sampled well enough by the "thermal" eccentricity distribution $f(e)=2 e$ (Ambartsumian 1937; Duquennoy \& Mayor 1991). For simplicity, we used the logarithmically flat distribution $d N / d \log a \propto a^{0}$ approach used by Metchev \& Hillenbrand (2009) to sample $a$. Thus, the joint prior probability is:

$$
P\left(\Psi \mid B_{n}\right) \propto q^{\beta}\left(\frac{2 e}{a}\right) \sin (i) .
$$

Table 4 shows the limits used in the simulations and corresponding prior for the orbital parameters. The sampling ranges for $i, e, T, \Omega$ and $\omega$ were taken by considering the total orbital plane with any orientation in space. More relevant for our detection limits, we focus our search region, mostly constrained by $a$, because the dynamics of binary-disk interaction models predict that tidal interaction between the binary star and the disk might truncate the inner region of the disk at radii of 2-3 times the semi-major axis of the binary orbit Artymowicz \& Lubow 1994). Table 6 shows the inner radii estimates that we used as limits to sample the semimajor axis space that ranges between $\frac{r_{d}}{2}$ and $\frac{r_{d}}{3}$ at distances of the corresponding star-forming region (Tau-Aur: 140 pc, Ophiuchus: $130 \mathrm{pc}$ and IC348: $220 \mathrm{pc}$ ).

Separately, Contrast ratio $(C)$ and Stellar Masses $\left(M_{1}, M_{2}\right)$ are sampled as a function of the corresponding distribution of the Mass ratio $(q)$, where these stellar masses together with the sample of semi-major values are used as inputs in Kepler's third law to compute the Orbital Periods ( $\mathrm{P}$, year) of each system. Here, we provided a brief summary of our methodology to sample $M_{1}, M_{2}$ and $C$.

- Stellar Masses and Contrast Ratios: Initially, we chose an isochrone $\left(Z_{1}\right)$ from the Baraffe et al. (2015) models, which represents the age of the TD and that in principle, plays the role of the primary component. In order to account for all the possible flux ratios, the following step is to start computing evolutionary tracks that describe the possible secondary stellar component in our data. This is done by randomly sampling a mass-ratio $\left(q_{n}\right)$ distribution
Table 4. Parameter Prior for Binary Model

\begin{tabular}{lccc}
\hline \hline \multicolumn{1}{c}{ Orbital Parameter } & Prior $^{1}$ & Lower Bound & Upper Bound \\
\hline Semi-major Axis (arc second) & Jeffreys' prior & $\frac{1}{3} R_{d}$ & $\frac{2}{3} R_{d}$ \\
Period (years) & Keplearian & $a_{i}^{3}$ & $a_{o}^{3}$ \\
Time of periastron passage (years) & Uniform & - & - \\
Eccentricity & $2 e$ & 0.01 & 0.9 \\
Inclination (degree) & sin (i) & 0 & 180 \\
Node (degree) & Uniform & 0 & 180 \\
Longitude of Periastron (degree) & Uniform & 0 & 360 \\
Mass-ratio & Power-Law & 0.01 & 1 \\
\hline${ }^{1}$ It was used the Cumulative Distribution Function to sample in the orbital parameter space.
\end{tabular}

and using the relation $Z_{2, n}=Z_{1} q_{n}$, where $Z_{2, n}$ and $Z_{1}$ are the tracks for all the possible secondary stars and the track for the primary star, respectively. Then, we computed the theoretical magnitudes of the these new evolutionary tracks by interpolating onto the theoretical stellar masses and $\mathrm{K}$ magnitudes of the chosen isochrone $\left(Z_{1}\right)$. Thus, we have generated a series of isochrones that correspond to every value of $q_{n}, q=0$ being a single star and $q=1$ a binary star system with similar masses. Once the evolutionary isochrones describing all the secondary components are computed and taking $Z_{1}$, we were able to calculate the total $\mathrm{K}$ magnitude of the binary system. Then, we estimated the primary mass $\left(M_{1}\right)$ based on its observed absolute $\mathrm{K}$ magnitude interpolated onto the total $\mathrm{K}$ magnitude of the binary system and the theoretical mass track; and with the relationship $M_{2}=q_{1, n} M_{1}$, we obtained the secondary mass, $M_{2}$. Finally, interpolating the primary and secondary masses onto the total $\mathrm{K}$ magnitude of the binary system and the theoretical mass track, we estimated their $\mathrm{K}$ magnitudes in order to compute contrast ratios.

Finally, the orbital parameters sampled are used to derive the angular separation $(\rho)$ and position angle $(\theta)$ (Meeus 1992) for $\mathrm{N}$ simulated binary systems. These values together with the calibrated data are used to compute the maximum likelihood of the contrast ratio and, thus confirm or rule out a stellar companion orbiting in the inner region of the disk.

3.1.1.2 Confirming a Single System: In the case of a single system as a point-symmetric target, the calibrated closure-phases are nearly equal to zero. Then, the computation of the global likelihood for a single system is basically determined by the conditional likelihood for a single system model with flux ratio equal to zero. From Equation 3 the global likelihood is:

$$
p\left(D \mid S_{n}\right)=p\left(C=0 \mid S_{n}\right)
$$

3.1.1.3 Odds Ratio: After computing the global likelihoods for $B_{n}$ and $S_{n}$, we are interested in comparing the two hypotheses, thus we computed the ratio of $p(B \mid D)$ and $p(S \mid D)$ known as Odds Ratio and written as $O_{B, S}$ in favor of the binary system model over the single system model:

$$
O_{B, S}=\frac{p(B \mid D)}{p(S \mid D)}=\frac{p\left(B_{n}\right)}{p\left(S_{n}\right)} \frac{p\left(D \mid B_{n}\right)}{p\left(D \mid S_{n}\right)}
$$

where the first factor on the right side of Equation 7 corresponds to the prior odds ratio equal to unity due to its uniformity in the parameter space and the second term is known as Bayes' Factor $(\Upsilon)$. Thus, $\Upsilon_{B, S}>>1$ means that a binary model is preferred by the data, $B_{B, S}<<1$ the data comes from a single star and $B_{B, S} \approx$ 
1 means that the odds are not modified and a binary and/or single star are equally probable.

\section{RESULTS}

We are considering visual companions to stars in our cluster down to a Ks magnitude of $\sim 15$ in IC348 (and significantly brighter limits in other clusters), where the stellar density is approximately $2 \times$ $10^{-4}$ stars per square arcsec at this magnitude limit. Given that we are only considering companions within $\sim 0.16$ arcsec in this paper, the probability of a background star masquerading as a physical companion is no more than $0.002 \%$ for each star in the sample, or $<0.04 \%$ for the entire sample.

As a part of our analysis and election of calibrator objects, we have found that 2MASS J04303235+3536133 is a binary object and although, it is reported as a TD by Cieza et al. (2010), we excluded this object from the sample because it is located at the Auriga-California molecular cloud. Table 5 shows contrast and positions for both observing epochs of the object.

\subsection{Stellar Companions Outside the Inner Radii}

In our first identification process, we identified LRL 72, LRL 182, LRL 213 and LRL 135 as cases for a high contrast detection with a confidence level of $\geq 99.5 \%$ (section 2). We removed those objects as a part of the sample of TDs because the stellar companion is located outside the area of study and is not responsible for carving out the inner region of the disk. Estimation of the companion mass values were obtained by taking the $K$ mag from the system, $\Delta K$ and Baraffe et al. (2015) models. Then, after obtaining an estimate of the $\mathrm{K}$ magnitudes for each stellar component, we interpolated linearly onto the evolutionary tracks to obtain the individual masses. Mass uncertainties are dominated by the evolutionary tracks, and depending on the theoretical model used, are of the order of 10$20 \%$ (Siess 2001). In the case of LRL 213, we included visibility amplitudes for breaking contrast/separation degeneracies. Table 5 shows the epoch, contrast ratio detection, position parameters and companion masses of these new binary systems.

The SEDs of these four close binary companions have NIR and MIR fluxes above the level expected for a reddened low mass star of spectral types M2-M5. The qualitative SEDs of these objects can be explained by a system composed of a close low-mass binary star, where the secondary component is surrounded by a "cold and weak" disk, a "circum-secondary disk". For instance, if the LRL 72 components are coeval and using the Baraffe et al. (2015) models, the object would correspond to a spectral type of M5-M7 with a temperature between 2900 and $3100 \mathrm{~K}$. As shown in Figure 4 the IR excess in the SED might be emitted by a disk orbiting the secondary component, instead of the primary star. Previous studies have shown that disks around brown dwarfs and very low mass stars are generally flatter and less massive than their counterpart T Tauri disks (Olofsson et al. 2013; Liu et al. 2015). This would explain the relative weak IR excesses observed in their SEDs of these binary systems.

Additionally, the angular separation of $\sim 100 \pm 0.4$ mas of LRL 72, imposes a limit on the extension of the disk of around 22 $\mathrm{au}$, because the presence of the companion at this close distance would truncate the disk. Similarly, LRL 182 and LRL 213 with a closer companions having mass values of $0.07 M_{\odot}$ at $\sim 7.7$ au and $0.15 M_{\odot}$ at $\sim 4.0 \mathrm{au}$, respectively, the disk also undergoes a faster dispersion as shown in its SED with a homogeneously small

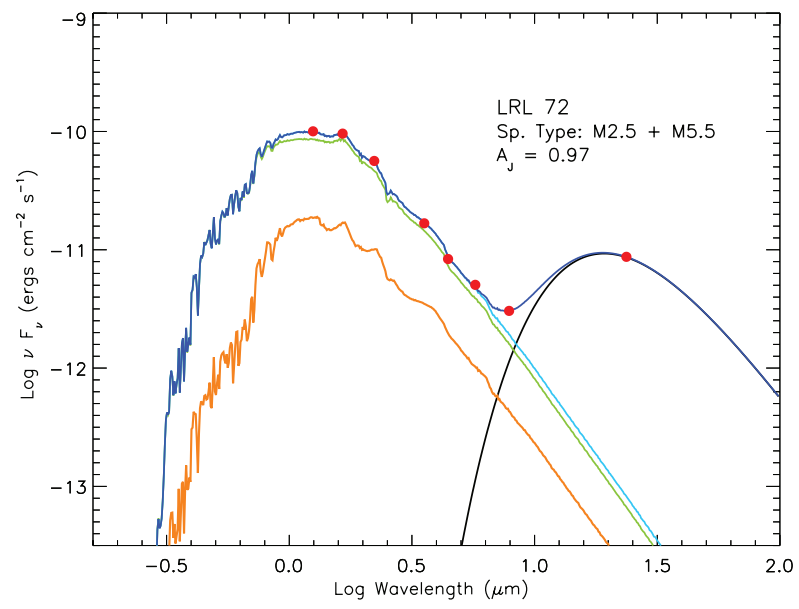

Figure 4: Spectral Energy Distribution for LRL 72 and its components with spectral types M2.5 and M5.5. Red dots show photometric data acquired from the literature, green and orange lines are the primary and secondary components BT-settl spectra, respectively. The black line is the disk black-body function values, the cyan line is the best stellar fit and the blue line is the sum of the primary and secondary spectra and the disk black-body function values.

IR excess, see Figure 3 For LRL135, the mass ratio is near unity $(q=0.89)$, and the system has a very weak excess with a disk (Figure 3). This system therefore remains a TD candidate, with it being unclear which component of the binary is the TD candidate. This system has a relatively high disk to stellar temperature ratio, with only a very small cleared inner disk region.

\subsection{Stellar Companions Inside the Inner Radii}

Our fits to closure phases showed detections of 7 new candidate companions. As shown in section 3 , we computed the Bayes' Factors for every object in the sample. Following the interpretation of Jeffreys (1998), we have found very strong Bayes' factors as an indication of the presence of candidate companions for LRL 31, $\mathrm{V} * \mathrm{~V}$ X-ray 6, WSB 12 and 2MASS J16335560-2442049 (see Table 6. For WSB 40, 2MASS J04210934+2750368 and 2MASS J16315473-2503238, we obtained moderate Bayes' factors (>10) from closure-phase alone, and considered these sources in more detail. In all cases, a visibility amplitude signal was found that was consistent with the best closure phase solution. In the case of WSB 40, the use of visibility amplitudes resulted in a clear solution with little degeneracy, as reported in Table 8 We assigned a high (>300) Bayes' factor to this object in Table 6 In the case of 2MASS J04210934+2750368, contrast ratio and separation were highly degenerate, so we list possible statistically significant solutions at plausible contrast ratios as shown in Table 9 For reported 2MASS J16315473-2503238, no binary solution was statistically significant (taken as $6 \sigma$, e.g. Kraus et al. 2016), and there was only 1 epoch on the target under variable Laser Guide Star conditions. For these reasons, we do not report a binary solution, but note that a binary companion was confirmed as a Double-Line Spectroscopic binary (SB2) star composed of a K7 and a K9, with a semi-major axis of $<0.6$ au by (Kohn et al. 2016) and therefore, we assigned a high (>300) Bayes' factor to this object in our statistical analysis (Table 6).

An interesting case is 2MASS J16335560-2442049, which 
Table 5. Companions Identified Outside the Inner Radii with the Aperture Mask

\begin{tabular}{|c|c|c|c|c|c|c|c|c|}
\hline Primary & $\begin{array}{c}\text { BJD } \\
(2400000+) \\
\end{array}$ & $\begin{array}{c}\Delta \mathbf{K} \\
{[\mathrm{mag}]}\end{array}$ & $\begin{array}{c}\text { Sep } \\
{[\mathrm{mas}]}\end{array}$ & $\begin{array}{l}\text { Sep } \\
{[\mathrm{au}]}\end{array}$ & $\begin{array}{c}\text { PA } \\
{[\text { deg.] }}\end{array}$ & $\begin{array}{c}\mathbf{M}_{1}{ }^{1} \\
{\left[\mathbf{M}_{\odot}\right]}\end{array}$ & $\begin{array}{c}\mathbf{M}_{2}{ }^{1} \\
{\left[\mathbf{M}_{\odot}\right]}\end{array}$ & Sig. ${ }^{2}$ \\
\hline LRL 72 & 55880.79 & $1.37 \pm 0.02$ & $103.72 \pm 0.36$ & $22.82 \pm 0.04$ & $221.86 \pm 0.10$ & 0.41 & 0.13 & 49.0 \\
\hline " & 56883.04 & $1.42 \pm 0.03$ & $100.45 \pm 0.47$ & $22.10 \pm 0.08$ & $226.70 \pm 0.26$ & $”$ & $"$ & 40.0 \\
\hline LRL 182 & 57000.75 & $1.54 \pm 0.04$ & $35.30 \pm 0.74$ & $7.76 \pm 0.26$ & $213.42 \pm 0.77$ & 0.20 & 0.05 & 39.4 \\
\hline LRL 135 & 57000.79 & $0.17 \pm 0.01$ & $82.01 \pm 0.26$ & $18.04 \pm 0.01$ & $208.36 \pm 0.21$ & 0.18 & 0.16 & 90.5 \\
\hline LRL 213 & 57000.83 & $0.57 \pm 0.18$ & $18.01 \pm 0.76$ & $3.96 \pm 0.17$ & $50.48 \pm 3.23$ & 0.11 & 0.06 & 9.30 \\
\hline $\mathrm{J} 04303235+3536133^{3}$ & 55880.96 & $0.40 \pm 0.36$ & $20.25 \pm 2.88$ & $9.11 \pm 1.30$ & $205.28 \pm 2.25$ & 0.72 & 0.59 & 10.31 \\
\hline$"$ & 57001.00 & $0.02 \pm 0.02$ & $26.24 \pm 0.70$ & $11.81 \pm 0.32$ & $216.81 \pm 2.19$ & 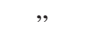 & $"$ & 9.48 \\
\hline
\end{tabular}

${ }^{1}$ The fractional uncertainties on the individual masses are $\leq 20 \%$.

${ }^{2}$ Significance in $\sigma$ is calculated as $\sqrt{\Delta \chi^{2} \times\left(N_{\mathrm{df}} / N_{\mathrm{cp}}\right)}$, with $N_{\mathrm{df}}$ the number of degrees of freedom and $N_{\mathrm{cp}}$ the number of closure-phases, and uncertainties scaled so that the reduced chi-squared of the best fit solution is unity.

${ }^{3}$ Located at Auriga-California molecular cloud ( $450 \pm 23$ pc; Lada, Lombardi \& Alves 2009).

Table 6. Bayesian Analysis

\begin{tabular}{lcccc}
\hline \hline \multicolumn{1}{c}{ Object } & Bayes' Factor & $\begin{array}{c}r_{d} \\
{[\mathbf{a u}]}\end{array}$ & $\begin{array}{c}r_{d} \\
{[\mathbf{m a s}]}\end{array}$ & $\begin{array}{c}\mathbf{T}_{d} \\
{[\mathbf{K}]}\end{array}$ \\
\hline \hline LRL 21 & 0.03 & $10.9 \pm 0.8$ & $49.0 \pm 4.0$ & $194.2 \pm 1.0$ \\
LRL 67 & 0.03 & $11.6 \pm 1.1$ & $53.0 \pm 5.0$ & $112.0 \pm 2.3$ \\
LRL 72 & - & $3.8 \pm 0.4$ & $17.0 \pm 2.0$ & $191.3 \pm 1.7$ \\
LRL 237 & 1.0 & $1.2 \pm 0.1$ & $5.0 \pm 0.4$ & $219.6 \pm 5.0$ \\
LRL 97 & 0.98 & $1.7 \pm 0.2$ & $8.0 \pm 1.0$ & $279.1 \pm 3.4$ \\
LRL 31 & 300 & $13.1 \pm 1.1$ & $60.0 \pm 5.0$ & $198.0 \pm 4.6$ \\
LRL 182 & - & $1.2 \pm 0.1$ & $6.0 \pm 0.4$ & $260.1 \pm 2.7$ \\
LRL 213 & - & $0.9 \pm 0.1$ & $4.0 \pm 0.4$ & $242.4 \pm 1.0$ \\
LRL 58 & 0.99 & $2.8 \pm 0.3$ & $13.0 \pm 1.0$ & $246.5 \pm 1.4$ \\
LRL 135 & 0.99 & $0.6 \pm 0.1$ & $2.0 \pm 0.4$ & $393.0 \pm 2.4$ \\
IRAS04125+2902 & 0.02 & $19.9 \pm 2.0$ & $143.0 \pm 14.0$ & $92.3 \pm 1.3$ \\
V410 X-ray 6 & $>300$ & $5.4 \pm 0.4$ & $39.0 \pm 2.0$ & $183.4 \pm 9.6$ \\
J04210934+2750368 & 24 & $10.5 \pm 1.2$ & $75.0 \pm 9.0$ & $91.0 \pm 1.9$ \\
EM* SR 24S & 0.01 & 29.0 & 223.0 & - \\
EM* SR 21A & 0.003 & $27.2 \pm 2.7$ & $209.0 \pm 21.0$ & $196.02 \pm 9.8$ \\
WSB 12 & $>300$ & $6.0 \pm 0.5$ & $46.0 \pm 4.0$ & $230.0 \pm 10.7$ \\
J16262367-2443138 & 0.07 & $8.3 \pm 0.7$ & $64.0 \pm 5.0$ & $211.0 \pm 2.3$ \\
J16273901-2358187 & 0.05 & $11.1 \pm 1.0$ & $85.0 \pm 7.0$ & $174.2 \pm 1.9$ \\
WSB 63 & 0.05 & $5.3 \pm 0.5$ & $41.0 \pm 4.0$ & $204.3 \pm 2.0$ \\
J16335560-2442049 & $>300$ & $7.3 \pm 0.7$ & $56.0 \pm 5.0$ & $178.2 \pm 3.3$ \\
J16250692-2350502 & 1.0 & $4.4 \pm 0.5$ & $35.0 \pm 4.0$ & $186.4 \pm 3.3$ \\
J16315473-2503238 & $>300$ & $6.2 \pm 0.6$ & $47.0 \pm 4.0$ & $266.4 \pm 11.5$ \\
WSB 40 & $>300$ & $6.7 \pm 0.6$ & $52.0 \pm 4.0$ & $209.8 \pm 1.4$ \\
V*V852 Oph & 0.05 & $16.2 \pm 2.1$ & $125.0 \pm 16.0$ & $93.1 \pm 3.6$ \\
\hline
\end{tabular}

${ }^{1}$ Targets not included in the statistical analysis of TDs.

${ }^{2}$ Estimated inner radius taken from Andrews et al. 2011).

${ }^{3}$ Based on Kohn et al. (2016). The Bayes' factor based on closure-phase alone was 30. See section 4.2 for a detailed discussion.

${ }^{4}$ Using visibility amplitude. The Bayes' factor based on closure-phase alone was 3. See section 4.2 for a detailed discussion.

was initially presented as a giant planet-forming candidate based on the morphology of its SED, large disk mass and modest accretion rate (Cieza et al. 2010; Orellana et al. 2012). Our $\chi^{2}$ minimization detected a secondary star located at $\sim 3.25 \pm 0.07$ au and using Baraffe et al. (2015) models and $\Delta \mathrm{K}$ mag, the stellar mass would correspond to $\sim 0.61 M_{\odot}$. However, the interpretation of a single-epoch for this object has to be taken with caution because of the high inclination $(\sim 50 \mathrm{deg})$ of its disk (Cieza et al. 2012a), and the known degeneracy between the contrast ratio and small angular separations in the NRM data (Pravdo et al. 2006). Recently, Cieza et al. (2013) demonstrated that the starlight scattered off the inner edge of the FL Tau disk could mimic the presence of a faint companion, which might be the case of 2MASS J16335560-2442049. Further observing epochs are needed to establish the physical origin of the non-zero closure phases found in our analysis. Other cases of new binary systems are WSB 40 and WSB 12, which were below the detection limits of Cheetham et al. (2015). Our careful reduction process and fits to closure phases resolved companions at $\sim$ $2.22 \pm 0.12$ au with a mass of $\sim 0.75 M_{\odot}$ for WSB 40 and at $\sim 2.60$ \pm 0.10 au with a mass of $\sim 0.75 M_{\odot}$ for WSB 12 . 
Table 7. Detection Confidence Limits (99.9\%)

\begin{tabular}{|c|c|c|c|c|c|c|}
\hline Object & $\begin{array}{c}\text { BJD } \\
(2400000+)\end{array}$ & $10-20$ & 20-40 & $\begin{array}{c}\Delta \mathbf{K}^{1} \\
\mathbf{4 0 - 8 0}\end{array}$ & $80-160$ & $160-240$ \\
\hline \multicolumn{7}{|c|}{ Non-Detections } \\
\hline LRL 21 & 55880.75 & 1.13 & 3.13 & 3.99 & 3.72 & 3.77 \\
\hline LRL 67 & 55880.83 & 1.83 & 3.59 & 4.44 & 4.18 & 4.24 \\
\hline LRL 237 & 56882.04 & - & 0.23 & 1.52 & 1.00 & 1.14 \\
\hline LRL 97 & 56882.04 & 0.59 & 2.75 & 3.80 & 3.54 & 3.57 \\
\hline LRL 58 & 57000.79 & 2.09 & 3.77 & 4.72 & 4.44 & 4.48 \\
\hline IRAS04125+2902 & 56881.12 & 1.60 & 3.41 & 4.29 & 4.04 & 4.09 \\
\hline EM* SR 24S & 54635.75 & - & 1.43 & 2.77 & 2.40 & 2.41 \\
\hline EM* SR 21A & 54635.88 & 2.51 & 4.11 & 4.98 & 4.71 & 4.75 \\
\hline J16262367-2443138 & 54635.88 & 2.28 & 3.91 & 4.81 & 4.57 & 4.58 \\
\hline J16273901-2358187 & 55674.96 & - & 0.44 & 2.01 & 1.60 & 1.67 \\
\hline WSB 63 & 55674.96 & 0.88 & 3.00 & 3.97 & 3.77 & 3.82 \\
\hline $\mathrm{J} 16250692-2350502$ & 55674.96 & - & 0.37 & 1.81 & 1.42 & 1.51 \\
\hline V*V852 Oph & 56032.08 & 1.60 & 3.41 & 4.31 & 4.06 & 4.12 \\
\hline \multicolumn{7}{|c|}{ Detections Outside Inner Radii } \\
\hline LRL 72 & 55880.79 & - & - & 0.55 & 0.31 & 0.38 \\
\hline ” & 56883.04 & - & - & 0.54 & 0.31 & 0.38 \\
\hline LRL 182 & 57000.75 & - & 0.13 & 1.08 & 0.70 & 0.80 \\
\hline LRL 135 & 57000.79 & - & - & 0.20 & - & 0.03 \\
\hline LRL 213 & 57000.83 & 0.68 & 2.85 & 3.83 & 3.63 & - \\
\hline \multicolumn{7}{|c|}{ Detections Inside Inner Radii } \\
\hline LRL 31 & 57000.79 & 0.93 & 3.02 & 3.91 & 3.72 & -0.00 \\
\hline V410 X-ray 6 & 55139.00 & 0.57 & 2.72 & 3.63 & 3.38 & 3.44 \\
\hline WSB 12 & 55675.00 & 0.56 & 2.69 & 3.63 & 3.37 & 3.41 \\
\hline WSB 40 & 56032.08 & 1.45 & 3.32 & 4.33 & 4.06 & 4.11 \\
\hline J16335560-2442049 & 55675.04 & 0.93 & 3.02 & 3.91 & 3.72 & - \\
\hline
\end{tabular}

NOTES: Angular separation ranges are given in mas.

${ }^{1}$ Limits within annuli.

Table 8. Companions Identified Inside the Inner Radii with the Aperture Mask

\begin{tabular}{lccccccc}
\hline \hline \multicolumn{1}{c}{ Primary } & $\begin{array}{c}\text { BJD } \\
(\mathbf{2 4 0 0 0 0 0 +})\end{array}$ & $\begin{array}{c}\Delta \mathbf{K} \\
{[\mathbf{m a g}]}\end{array}$ & $\begin{array}{c}\text { Sep } \\
{[\mathbf{m a s}]}\end{array}$ & $\begin{array}{c}\text { Sep } \\
{[\mathbf{a u}]}\end{array}$ & $\begin{array}{c}\text { PA } \\
{[\mathbf{d e g} .]}\end{array}$ & $\begin{array}{c}\mathbf{M}_{2}{ }^{1} \\
{\left[\mathbf{M}_{\odot}\right]}\end{array}$ & Sig. \\
\hline \hline LRL 31 & 57000.92 & $3.92 \pm 0.20$ & $38.09 \pm 5.30$ & $8.38 \pm 1.16$ & $45.56 \pm 4.06$ & 0.20 & 6.70 \\
V410 X-ray 6 & 55138.92 & $0.15 \pm 0.07$ & $22.96 \pm 1.25$ & $3.22 \pm 0.18$ & $87.80 \pm 2.20$ & 0.21 & 12.50 \\
WSB 12 & 55674.92 & $0.42 \pm 0.11$ & $20.29 \pm 0.78$ & $2.64 \pm 0.10$ & $351.02 \pm 2.13$ & 0.75 & 11.80 \\
WSB 40 & 56031.51 & $0.35 \pm 0.14$ & $17.42 \pm 0.94$ & $2.26 \pm 1.12$ & $-11.37 \pm 3.80$ & 0.75 & 8.40 \\
J16335560-2442049 & 55675.04 & $0.10 \pm 0.05$ & $25.30 \pm 0.55$ & $3.29 \pm 0.71$ & $344.55 \pm 1.77$ & 0.61 & 14.40 \\
\hline
\end{tabular}

NOTES: Significance in $\sigma$ is calculated as $\sqrt{\Delta \chi^{2} \times\left(N_{\mathrm{df}} / N_{\mathrm{cp}}\right)}$, with $N_{\mathrm{df}}$ the number of degrees of freedom and $N_{\mathrm{cp}}$ the number of closure-phases, and uncertainties scaled so that the reduced chi-squared of the best fit solution is unity.

${ }^{1}$ The fractional uncertainties on the stellar masses are $\leq 20 \%$.

Table 9. Degenerate Companion Solutions for 2MASS J04210934+2750368 at $\Delta \mathrm{K} 0.5,1,2$ and 3.

\begin{tabular}{cccccccc}
\hline \hline Primary & $\begin{array}{c}\text { BJD } \\
(\mathbf{2 4 0 0 0 0 0 +})\end{array}$ & $\begin{array}{c}\Delta \mathbf{K} \\
{[\mathbf{m a g}]}\end{array}$ & $\begin{array}{c}\text { Sep } \\
{[\mathbf{m a s}]}\end{array}$ & $\begin{array}{c}\text { Sep } \\
{[\mathbf{a u}]}\end{array}$ & $\begin{array}{c}\text { PA } \\
{[\mathbf{d e g} .]}\end{array}$ & $\begin{array}{c}\mathbf{M}_{2} \\
{\left[\mathbf{M}_{\odot}\right]}\end{array}$ & Sig. \\
\hline \hline J04210934+2750368 & 57001.04 & 0.5 & $19.28 \pm 0.69$ & $2.70 \pm 0.09$ & $309.60 \pm 3.60$ & 0.09 & 10.25 \\
$"$ & $"$ & 1.0 & $19.01 \pm 0.75$ & $2.66 \pm 0.10$ & $307.68 \pm 3.92$ & 0.07 & 9.87 \\
$"$ & $"$ & 2.0 & $21.78 \pm 1.32$ & $3.05 \pm 0.18$ & $306.30 \pm 4.63$ & 0.04 & 8.54 \\
$"$ & $"$ & 3.0 & $26.40 \pm 3.13$ & $3.70 \pm 0.94$ & $306.89 \pm 6.78$ & 0.02 & 6.74 \\
\hline
\end{tabular}




\subsection{Stars without a companion in the Inner Radii}

With a Bayes' factor of $<0.1$, the NRM data analysis did not detect binary stars with angular separations ranging from $\sim \frac{1}{3}$ to $\frac{2}{3}$ of the $r_{d}$ for LRL 21, LRL 67, IRAS04125+2902, EM* SR 21A, DoAr 44, 2MASS J16262367-2443138, 2MASS J162739012358187, WSB 63, EM* SR 24S and V*V852 Oph (Table 6). Detection limits for these objects are listed in Table 7 The absence of a binary companion implies that the inner region mainly is being dispersed by an internal process that determines the lifetime of the disk. To date, the different mechanisms proposed to explain the inner holes of these disks do not accurately predict the observed features of TDs, and produced theoretically distinct properties of the TDs (e.g. Alexander \& Armitage 2007). Despite all the efforts to develop a unique explanation of the evolution of the disk and its transition phase from a class II to III, photoevaporation and planetary formation, and its counterpart processes such as dust filtration and grain growth, seem to be the most efficient mechanisms to disperse the disk from the inside out.

\subsection{Unresolved Transitional Disks}

In Table 6 LRL 237, LRL 97, LRL 58 and 2MASS J162506922350502 are the objects with Bayes' factors of $\sim 1$, meaning that our NRM observations were not able to rule out or confirm companions for those objects, where the inner radii estimations fall inside our detection limit of 25 mas. These objects have M1 or later spectral types, meaning that they are at the low mass end of our sample. They also have relatively low accretion rates, typical of lower mass objects (Herczeg \& Hillenbrand 2008, $\lesssim 10^{-8} M_{\odot} \mathrm{yr}^{-1}$ ) . If their lack of a NIR excess is due to clearing by a binary companion, such a companion can only be discovered by multi-epoch radial velocity monitoring. We summarise our detection limits in Table 7

\section{STATISTICS}

After estimating the stellar properties and computing the Bayes' factor $\Upsilon_{B, S}=\frac{P(D \mid B)}{P(D \mid S)}$ for every target, we proceed to estimate the frequency of binary stars producing the "transitional disk SEDs".

Continuing with Bayesian statistics, we assigned prior probabilities to each population, where the prior information for a binary or single system in TDs are equally probable. A non-informative probability distribution for the frequency of binary or single systems is a parameter representing two unique scenarios, binary or non-binary. Thus, $P(B)=\gamma$ represents the probability of binarity and $P(S)=(1-\gamma)$ not binarity, where the paramenter $\gamma \in[0,1]$ and is sampled by the family of beta distributions ${ }^{3}$ (Glickman \& $\operatorname{van}$ 2007).

The Jeffreys prior for the sampling distribution that provides uniform probability to both events B and $\mathrm{S}$ is represented by:

$$
P(\gamma) \propto \frac{1}{\sqrt{\gamma(1-\gamma)}} \propto \frac{1}{\sqrt{P(B) P(S)}} .
$$

Taking into account that the current work is pioneering in the search for close binary companions (> 40 mas) or confirming a single star in the inner region of TDs, no information was available

\footnotetext{
${ }^{3}$ Beta distribution has parameters $\alpha, \beta=1 / 2$ to ensure a probability equal to unity and events equally probable.
}

from inside the inner region of these TDs. Therefore, we have to assume that the data have arisen from one of two systems and being equally probable. Thus, a prior distribution $\gamma$ with the form that represents the best prior state of knowledge can be modified with the observed data as follows:

$$
P(\gamma \mid D) \propto P(\gamma) P(D \mid \gamma) \propto P(\gamma)\left[\gamma\left(\Upsilon_{B, S}\right)+(1-\gamma)\right]
$$

where $\Upsilon_{B, S}$ is the computed Bayes' factor of every TD. Considering all TDs in our sample and additionally TDs from previous studies, the posterior density function of the frequency of binary systems responsible of the observed SEDs is:

$$
P(\gamma \mid D) \propto P(\gamma) \prod_{i=1}^{i}\left[\gamma\left(\Upsilon_{B, S}\right)_{i}+(1-\gamma)\right]
$$

with the index $i$ representing the number of objects included in the modification of $\gamma$.

\subsection{Binary Frequency in TDs}

Binary and single objects were identified in our sample of TDs with Bayes' factors $\Upsilon<0.1, \Upsilon>300$ and $\Upsilon \simeq 1.0$, which are likely single stars, binary stars or unresolved systems, respectively, except for 2MASS J04210934+2750368 with a Bayes' factor of 24 (Table 6); we proceeded to include objects that were already classified as TDs and eventually, were characterized as binary or single star 4. For these binary and single objects a Bayes' factor of 300 and 0.001 were used in the Bayesian statistical analysis, respectively. These objects are summarised in Table 10 After computing the posterior probability, see Equation 10 our uniform prior has been modified to the posterior probability of $0.38 \pm 0.09$ and shown in Figure 5

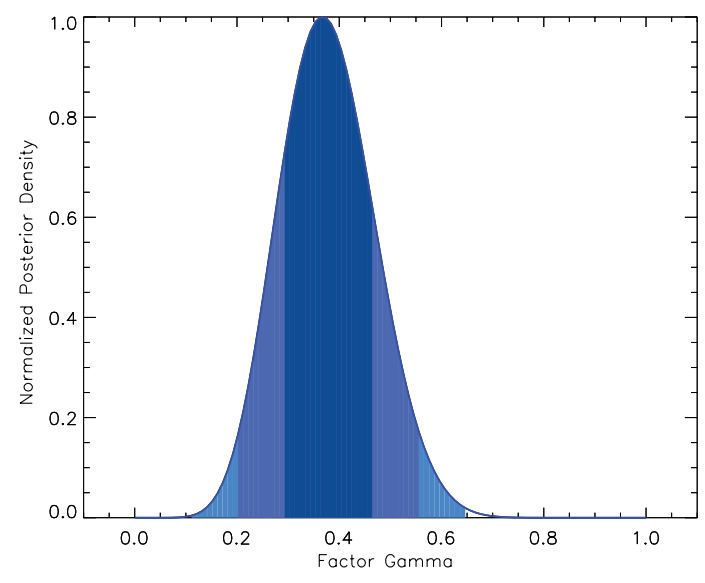

Figure 5: The fraction of TD in our sample consistent with a binary system being the main mechanism causing the characteristic SED is $0.38 \pm 0.09$.

\footnotetext{
${ }^{4}$ For the special cases of 2MASS J16315473-2503238 and previously studied, FL Cha, we opted to include them as binary objects in the statistical analysis.
} 
Table 10. Stellar Properties of Other Known TDs

\begin{tabular}{lccccccc}
\hline \hline \multicolumn{1}{c}{ Object } & R.A. (J2000) & Dec. (J2000) & Type & Spec. Type & Accretor & $\begin{array}{c}\mathbf{R}_{c a v .} \\
\text { [au] }\end{array}$ & Reference \\
\hline \hline Coku Tauri/4 & 044116.808 & +284000.07 & Binary & M1.5 & $\mathrm{n}$ & 10 & $1,2,11$ \\
LKCa15 & 043917.796 & +222103.48 & Single & K5v & y & 50 & $1,3,5,9$ \\
DM Tau & 043348.73 & +181010.0 & Single & M1 & y & 19 & $1,4,5,9$ \\
GM Aur & 045510.983 & +302159.54 & Single & K5 & y & 28 & $1,4,5,9$ \\
UX Tau A & 043003.988 & +181349.61 & Single & G8 & y & 25 & $1,4,5,9$ \\
RY Tau & 042157.41 & +282635.57 & Single & K1 & y & 18 & $1,5,10$ \\
CS Cha & 110224.912 & -773335.72 & Binary & K6 & y & 38 & $1,6,10$ \\
T Cha & 115713.550 & -792131.54 & Single & K0 & y & 40 & $1,6,12$ \\
FL Cha & 110839.051 & -771604.24 & Binary & K8 & y & 8.3 & 1,7 \\
TW Hydrae & 110151.907 & -344217.03 & Single & K6 & y & 41 & $1,8,13$ \\
Haro 1-16 & 163133.46 & -242737.3 & Single & K3 & y & 36 & $1,14,15$ \\
\hline
\end{tabular}

Reference: (1) 2MASS All-Sky Point Source Catalog, (2) Ireland \& Kraus (2008), (3) Kraus et al. (2012), (4) Huélamo et al. (2011), (5) Pott et al. (2010), (6) Guenther et al. (2007), (7) Cieza et al. (2013), (8) Rapson et al. (2015), (9) Andrews et al. (2011), (10) Espaillat et al. (2011), (11) D’Alessio et al. (2005), (12) Huélamo et al. (2015), (13) Nomura et al. (2016), (14) Bouvier \& Appenzeller (1992), (12) Cheetham et al. (2015)

\section{DISCUSSION}

In our combined sample consisting of 31 objects, including 11 TDs and CDs with known multiplicity from the literature, and excluding 3 wide binaries, we find that a fraction of $0.38 \pm 0.09$ of the SEDs are being produced by the flux emission of a binary star + disk instead of a single star + disk. This means that the remaining SEDs with low NIR and MIR excesses observed to date are the result of the dispersion of the primordial material due to another internal mechanism. Our binary detections inside the fitted disk wall inner radii do not necessarily have projected separations between $\frac{1}{3}$ and $\frac{1}{2}$ of the inner radii, which is the expected semi-major axis range for a binary to cause the truncation of the disk. However, all detections lie within $\frac{1}{2}$ of our calculated inner disk radii, consistent with projection effects.

Given the criteria applied to select our sample and following the standards for disk classification, we emphasize that these objects should be treated as CDs that possibly are in a transitional phase, and no longer treat them as TDs with a single star. Originally, the SEDs of these objects were described assuming only one object in the interior of the disk and using detailed disk models to fit the excess continua (e.g. Espaillat et al. 2012). As demonstrated by this work, there is a significant fraction of these SEDs which were mis-classified. However, as seen in Figure 3 the CD SEDs of LRL 31, V410 X-ray 6, WSB 12, WSB 40 , 2MASS J163355602442049, 2MASS J04210934+2750368 and 2MASS J163154732503238 are indistinguishable from TDs. Although, to date the resemblance between CD SEDs and TD SEDs is well established (e.g. Ireland \& Kraus 2008), unfortunately we could not set an observational constraint such as accretion rate or flux emission in our sample. For example, the SEDs of V410 X-ray 6 and 2MASS J16335560-2442049 bear a resemblance to the large MIR emission and zero NIR excess detected in the binary Coku Tau/4 (D'Alessio et al. 2005; Ireland \& Kraus 2008), while the other objects show a more similar SED to a typical TD SED. On the other hand, for those objects shown in Table 6 with Bayes' factors $\simeq 1$, that due to resolution limitations we were not able to confirm or rule out their binarity, multi-epoch RV monitoring observations are needed (e.g. Kohn et al. 2016), because there might be more binary objects dispersing the inner region of the disk efficiently.

We have also detected 4 new binary systems with the location of the secondary component outside the inner region of the disk. In- terestingly, these systems produced SEDs characteristic of the TDs and are low accretors (Table 5. We have proposed that those SEDs composed of a low-mass binary star with one of its components orbiting outside the inner radius of the disk, might have its more "evolved" disk orbiting the sub-stellar companion, instead of the primary component. Although, it is also plausible that the primary component has a circumstellar disk that is being dispersed by the close sub-stellar companion. Previously, Harris et al. (2012) performed a high angular resolution millimeter-wave dust continuum imaging survey of circumstellar material associated with the individual components of multiple star systems in the Taurus-Auriga young cluster. They found that the presence of a close stellar companion $(<30 \mathrm{au})$ impacts disk properties, producing a disk mass depletion with a factor of $\sim 25$. In the case of the LRL 72, LRL 182, LRL 213 and LRL 135 systems, a faster dispersion of the disk by the presence of the stellar companion located at $\leq 20$ au could influence the initial conditions for the formation of planets and prevent the first steps of this evolutionary process (e.g. dust settling and grain growth).

\subsection{Physical Sources of Typical TD SEDs}

Planetary formation could potentially explain the estimated inner optically thick disk radii for these objects and therefore, the peculiar shape or decreased flux observed in the NIR/MIR SEDs of these TDs. Depending on the inner hole size, the gap could be cleared up by single or multiple planets orbiting this region Lubow, Seibert \& Artymowicz 1999; Rice et al. 2006b; DodsonRobinson \& Salyk 2011). In the context of planet disk interaction, and as a consequence of a massive planet clearing out the inner region of the disk, a local pressure bump is created at the inner edge of the outer disk. In the last decade, this local pressure bump was proposed to act as a filter at the outer edge of a disk gap, filtering particles of size $\gtrsim 10 \mu \mathrm{m}$ and impeding the drift inward of them (Rice et al. 2006a). As a result of this dust filtration, the disk profile is shown with an abrupt discontinuity in its dust radial profile and at the same time permits the presence of small particles closer to the central star $(\lesssim 10 \mu \mathrm{m})$ (e.g Garufi et al. 2013). Thus, this optically thin dust might be responsible of the weak NIR/MIR excess present in TD SEDs. In addition, inside this cavity coupling between $\mu \mathrm{m}$ size dust grains and gas is expected (Garufi et al. 2013), 
while the location to pile-up the dust at a sub- to millimeter scale in a pressure maximum, leads to different locations of gap edges for gas and "bigger" dust particles (Pinilla, Benisty \& Birnstiel 2012). In our approach to estimate inner cavities, we consider the location of particles of $\sim 0.1 \mu \mathrm{m}$ that might coincide with the gaseous cavity, ingredients necessary to explain the detected accretion rates in our sample of TDs.

Most of our TDs show accretion rates ranging from $10^{-8}$ to $10^{-10} M_{\odot} / \mathrm{yr}$ and, although these accreting TDs are also ideal targets to test the role of some photoevaporation models (e.g. Alexander, Clarke \& Pringle 2006a b), there are other missing pieces to the puzzle such as disk mass measurements needed to obtain a complete picture of this transitional phase. Therefore, the observed SEDs of TDs with the presence of a single star might be subject to a dominating internal mechanism and the amount of mass in the disk. Thus, in order to distinguish the dominating dispersal mechanism producing the inner holes in the disks, a follow-up program of millimeter observations of the TDs is required to be able to estimate the disk mass of these objects. Nevertheless, the inner region of these TDs could be depleted by a combination of two or more mechanisms that dominate at different distances from the central star and timescales dictated by the initial physical conditions.

\subsection{Single vs. Binary Stars: Hosting Planetary Formation}

At first glance, it is tempting to suggest that single stars have a higher probability of hosting the formation of planetary systems than close binary systems. However, Pascucci et al. (2008) studied the first steps of planetary formation in single and binary systems with projected separations between $\sim 10$ and 450 au and they found no statistical significant difference in the degree of dust settling and grain growth of those systems, indicating that expected differences in the exoplanet properties arise in the later stages of their formation and/or migration (e.g. Kley 2000; Kley \& Nelson 2007). Our close binary companions are detected at angular separations between $2-10 \mathrm{au}$; these small angular separations might affect the initial conditions for the formation of planets in the inner region of the circumbinary disks. This is mainly due to the modification of the binary eccentricity and excitation of density waves generated by the resonant interactions of the binaries with the disk, which remove primordial material (Lubow \& Artymowicz 2000). Based on these assumptions, the "weak" excess from the circumstellar material in the SEDs of the CDs, increased by the secondary flux radiation, could point out a lower probability for the formation of a planet in radii of around $a \leq 10$ au in very close binary stars. On the other hand, single stars are more probable to host forming planets at inner radii around $<10$ au than close binary stars, where actually most of the planet formation might take place.

Because the time available to form any planet(s) in a circumstellar disk might vary depending on the initial conditions and the evolution of the disk, it is necessary in future surveys to characterize the distribution of disk masses in CDs with close binary and single stars, that together with the accretion rates will establish the physical parameters constraining where and when planets form in those systems. Additionally, accretion rates have been used to estimate the dissipation of the primordial disks once accretion stops; however, we did not find any trend in $\dot{M}_{*}$ or difference between close binary and single stars in our sample that helps us to constrain the timescales of these systems.

\section{SUMMARY}

Using infrared NRM interferometry taken with the Keck II telescope, we have observed a sample of 24 TDs located in the TaurusAuriga, IC-348 and Ophiuchus star-forming regions. We implemented a new method of completeness correction for our detection limits, which combines randomly sampled binary orbits and Bayesian inference. With high confidence levels of $99 \%$, a total of 7 close binary candidates have been detected orbiting the inner radii of the TDs, and likely being the main mechanism responsible for the dispersion inside out the inner disk. Also, we found four binary companions orbiting outside the inner radii of their TDs and we have suggested that the unusual SEDs of these systems are due to a disk orbiting a substellar secondary companion, producing similar SEDs as the single and/or close binary stars surrounded by a more "evolved" disk or weak disk.

Including 11 known TDs from the literature and whose binarity was already confirmed or ruled out, we have a total of 31 TDs that are part of our Bayesian analysis (Section 5). Updating a uniform prior distribution, we obtained a significant fraction of $0.38 \pm$ 0.09 objects with TD SEDs that are actually CDs. This fraction represents the unusual SEDs with a lack of excess in the NIR and/or MIR being produced by the flux emission of a close-binary companion and a disk. This fraction must be taken into consideration for future surveys and studies of these transitional objects in order to decode the disk evolution process and the timescales of close binary and single stars, separately. The remaining SEDs are being produced by a single system and a disk in a transition phase, where the main cause of dispersion could be any other internal mechanism such as photoevaporation, grain growth and/or planet disk interactions.

\section{ACKNOWLEDGEMENTS}

We thank the referee for her/his comments and suggestions, which helped in the improvement of the paper. M.I was suported by the Australian Research Council's Future Fellowship scheme (FT130100235). L.A.C. acknowledges support from the Millennium Science Initiative (Chilean Ministry of Economy), through grant ÒNucleus RC130007Ó. L.A.C. was also supported by CONICYT-FONDECYT grant number 1140109.

\section{APPENDIX A: DISTANCE TO IC348 REGION}

The distance to the Perseus molecular cloud has been measured in a wide range between 220 - 380 pc (Harris, Morgan \& Roman 1954; Herbig \& Jones 1983; Cernis 1993; Scholz et al. 1999; de Zeeuw et al. 1999; Hirota et al. 2008; Hirota 2010), and choosing the most appropriate measurement must be taken with caution because of the influence on other observational estimations, such as age and luminosity of the targets. For our purposes, we base our decision to adopt a distance to IC 348 on the astrometric observations of $\mathrm{H}_{2} \mathrm{O}$ maser sources by Hirota (2010). They used the VERA longbaseline array to estimate a distance to SVS 13 in the NGC 1333 cluster of $235 \pm 18 \mathrm{pc}$ and a distance of $232 \pm 18 \mathrm{pc}$ to L1448. In addition, Sun et al. (2006) mapped the Perseus molecular cloud complex simultaneously in ${ }^{13} \mathrm{CO}(\mathrm{J}=2-1)$ and ${ }^{12} \mathrm{CO}(\mathrm{J}=2-1)$ using the KOSMA 3 m submillimeter telescope. They found a dynamical connection with a velocity gradient between NGC 1333 at $\sim 7 \mathrm{~km}$ $s^{-1}$, L 1448 at $\sim 8 \mathrm{~km} \mathrm{~s}^{-1}$ and IC 348 at $\sim 9 \mathrm{~km} \mathrm{~s}^{-1}$ within a diameter of $20 \mathrm{pc}$ of extension. Considering that the Perseus molecular 
Table A1. IC348 1 Properties

\begin{tabular}{lc}
\hline \multicolumn{1}{c}{ Property } & Value \\
\hline \hline R.A. $(\mathrm{J} 2000)^{1}$ & $03: 44: 56.15$ \\
Dec. $(\mathrm{J} 2000)^{1}$ & $+32: 09: 15.5$ \\
Spec. Type & $\mathrm{B} 5$ \\
$L_{*}\left(L_{\odot}\right)^{2}$ & 324.8 \\
$A_{J}{ }^{3}$ & 0.55 \\
$T_{*}(\mathrm{~K})$ & 15400 \\
$M_{*}\left(M_{\odot}\right)$ & 4.6 \\
Dist. $(\mathrm{pc})$ & 220 \\
\hline
\end{tabular}

Reference: (1) 2MASS All-Sky Point Source Catalog, (2) Luminosity estimated following the method from Kenyon \& Hartmann (1995), (3) $\mathrm{A}_{J}$ estimated as described in Section 2.1 (4) Spectral type and stellar temperature were adopted from Luhman et al. (2003).

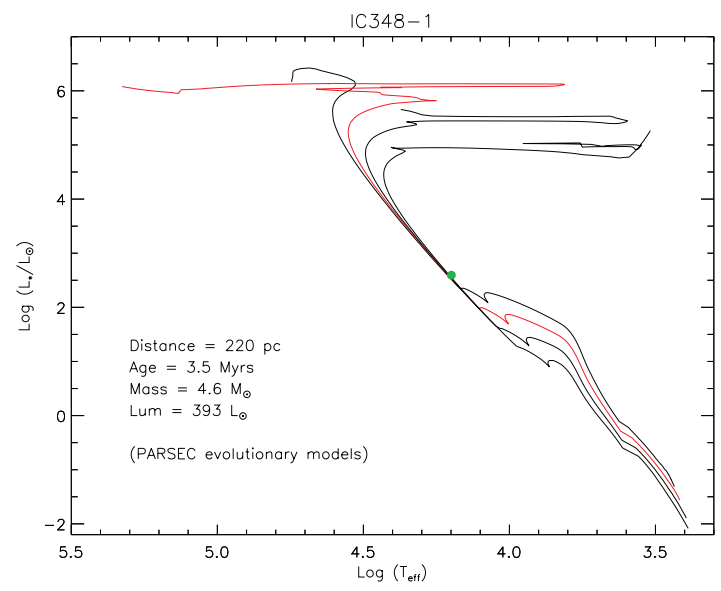

Figure A1: PARSEC evolutionary models for young stars (Bressan et al. 2012). Black Solid lines in descending order are 2, 3.5, 6 and 10 Myrs isochrones. Red solid line corresponds to the 3.5 Myrs isochrone used to characterised IC348-1 and then, estimate a distance to the IC348 star-forming region.

cloud (containing IC348, NGC1333, L1448, L1445; Hirota 2010) has a full angular extent of only $\pm 3^{\circ}$ from its centre, it is expected to have a characteristic distance range of $5 \%$.

Here, we use the most luminous member of the cluster, IC 348 LRL 1, composed of two stars of similar brightness with spectral types B5V to adopt an independent distance to IC348 objects. IC 348 LRL 1 is a binary system with an angular separation of 0.47 " and P.A. of $17.9^{\circ}$ Alzner 1998) and as the only member of B spectral type in the region, it can be used to estimate a distance to it (Luhman et al. 1998). At early ages, $\leq 10$ Myrs, the bolometric luminosity of these B spectral type massive stars do not vary significantly, giving an independence of the isochrone used to describe the target. This allows a spectral type conversion into effective temperature without constraints on the age of the system. Figure A1 presents the isochrones of 2, 3.5, 6 and 10 Myrs, as they are the most representative to describe young members of IC348. Clearly, the isochrones used to predict the stellar parameters for IC 348 LRL 1 are mostly invariant at this early stage of the stellar evolution.

We derived the stellar properties of IC 348 LRL 1 based on the spectral type and a conversion to the stellar temperature, see Table A1 According to the PARSEC evolutionary models (Bressan et al. 2012), a B5V object with a stellar mass of $4.2 \mathrm{M}_{\odot}$ should be located at a distance of $220 \pm 10 \mathrm{pc}$, see Figure A1 Thus, a stellar luminosity of $393 \mathrm{~L}_{\odot}$ is calculated with the dereddened Jband photometry method from Kenyon \& Hartmann (1995) and $A_{J}$ extinction was estimated as explained in Section 2.1 


\section{REFERENCES}

Alexander R. D., Armitage P. J., 2007, MNRAS, 375, 500

Alexander R. D., Clarke C. J., Pringle J. E., 2006a, MNRAS, 369, 216

Alexander R. D., Clarke C. J., Pringle J. E., 2006b, MNRAS, 369, 229

Allard F., 2014, in IAU Symposium, Vol. 299, IAU Symposium, Booth M., Matthews B. C., Graham J. R., eds., pp. 271-272 Alzner A., 1998, A\&AS, 132, 237

Ambartsumian V. A., 1937, Astron. Zh., 14, 207

Andrews S. M., Wilner D. J., Espaillat C., Hughes A. M., Dullemond C. P., McClure M. K., Qi C., Brown J. M., 2011, ApJ, 732, 42

Artymowicz P., Lubow S. H., 1994, ApJ, 421, 651

Baraffe I., Homeier D., Allard F., Chabrier G., 2015, A\&A, 577, A42

Bayo A., Rodrigo C., Barrado Y Navascués D., Solano E., Gutiérrez R., Morales-Calderón M., Allard F., 2008, A\&A, 492, 277

Bouvier J., Appenzeller I., 1992, A\&AS, 92, 481

Bressan A., Marigo P., Girardi L., Salasnich B., Dal Cero C., Rubele S., Nanni A., 2012, MNRAS, 427, 127

Canovas H., Caceres C., Schreiber M. R., Hardy A., Cieza L., Ménard F., Hales A., 2016, ArXiv e-prints

Cernis K., 1993, Baltic Astronomy, 2, 214

Cheetham A. C., Kraus A. L., Ireland M. J., Cieza L., Rizzuto A. C., Tuthill P. G., 2015, ApJ, 813, 83

Chiang E., Murray-Clay R., 2007, Nature Physics, 3, 604

Cieza L. A. et al., 2013, ApJL, 762, L12

Cieza L. A. et al., 2012a, ApJ, 752, 75

Cieza L. A. et al., 2010, ApJ, 712, 925

Cieza L. A., Schreiber M. R., Romero G. A., Williams J. P., Rebassa-Mansergas A., Merín B., 2012b, ApJ, 750, 157

Clarke C. J., Gendrin A., Sotomayor M., 2001, MNRAS, 328, 485

Currie T., Kenyon S. J., 2009, AJ, 138, 703

Cutri R. M. et al., 2003, VizieR Online Data Catalog, 2246

D'Alessio P. et al., 2005, ApJ, 621, 461

de Zeeuw P. T., Hoogerwerf R., de Bruijne J. H. J., Brown A. G. A., Blaauw A., 1999, AJ, 117, 354

Dodson-Robinson S. E., Salyk C., 2011, ApJ, 738, 131

Duchêne G., Ghez A. M., McCabe C., Weinberger A. J., 2003, ApJ, 592, 288

Dullemond C. P., Dominik C., Natta A., 2001, ApJ, 560, 957

Dullemond C. P., Monnier J. D., 2010, ARA\&A, 48, 205

Duquennoy A., Mayor M., 1991, A\&A, 248, 485

Espaillat C., Andrews S., Powell D., Feldman D., Qi C., Wilner D., DAlessio P., 2015, ApJ, 807, 156

Espaillat C., Calvet N., D’Alessio P., Hernández J., Qi C., Hartmann L., Furlan E., Watson D. M., 2007, ApJL, 670, L135

Espaillat C. et al., 2010, ApJ, 717, 441

Espaillat C., Furlan E., D’Alessio P., Sargent B., Nagel E., Calvet N., Watson D. M., Muzerolle J., 2011, ApJ, 728, 49

Espaillat C. et al., 2012, ApJ, 747, 103

Espaillat C. et al., 2014, Protostars and Planets VI, 497

Evans, II N. J. et al., 2003, PASP, 115, 965

Evans, II N. J. et al., 2009, ApJS, 181, 321

Flaherty K. M., Muzerolle J., Rieke G., Gutermuth R., Balog Z.,

Herbst W., Megeath S. T., Kun M., 2011, ApJ, 732, 83

Forrest W. J. et al., 2004, ApJS, 154, 443

Furlan E. et al., 2011, ApJS, 195, 3

Garufi A. et al., 2013, A\&A, 560, A105

Glickman M., van D. D., 2007, Methods Mol Biol, 404, 319
Guenther E. W., Esposito M., Mundt R., Covino E., Alcalá J. M., Cusano F., Stecklum B., 2007, A\&A, 467, 1147

Harris D. L., Morgan W. W., Roman N. G., 1954, ApJ, 119, 622

Harris R. J., Andrews S. M., Wilner D. J., Kraus A. L., 2012, ApJ, 751,115

Hartmann L., 2001, AJ, 121, 1030

Herbig G. H., Jones B. F., 1983, AJ, 88, 1040

Herczeg G. J., Hillenbrand L. A., 2008, ApJ, 681, 594

Hirota T., 2010, in 10th European VLBI Network Symposium and EVN Users Meeting: VLBI and the New Generation of Radio Arrays, p. 63

Hirota T. et al., 2008, PASJ, 60, 37

Huélamo N., de Gregorio-Monsalvo I., Macias E., Pinte C., Ireland M., Tuthill P., Lacour S., 2015, A\&A, 575, L5

Huélamo N., Lacour S., Tuthill P., Ireland M., Kraus A., Chauvin G., 2011, A\&A, 528, L7

Ireland M. J., Kraus A., Martinache F., Lloyd J. P., Tuthill P. G., 2008, ApJ, 678, 463

Ireland M. J., Kraus A. L., 2008, ApJL, 678, L59

Isella A., Natta A., 2005, A\&A, 438, 899

Janson M. et al., 2012, ApJ, 754, 44

Jeffreys H., 1998, Theory of probability, Oxford Classic Texts in the Physical Sciences. The Clarendon Press, Oxford University Press, New York, pp. xii+459, reprint of the 1983 edition

Kenyon S. J., Hartmann L., 1995, ApJS, 101, 117

Kley W., 2000, in IAU Symposium, Vol. 200, IAU Symposium, p. 211

Kley W., Nelson R., 2007, ArXiv e-prints

Koepferl C. M., Ercolano B., Dale J., Teixeira P. S., Ratzka T., Spezzi L., 2013, MNRAS, 428, 3327

Kohn S. A., Shkolnik E. L., Weinberger A. J., Carlberg J. K., Llama J., 2016, ApJ, 820, 2

Kraus A. L., Andrews S. M., Bowler B. P., Herczeg G., Ireland M. J., Liu M. C., Metchev S., Cruz K. L., 2015, ApJL, 798, L23 Kraus A. L., Ireland M. J., Hillenbrand L. A., Martinache F., 2012, ApJ, 745, 19

Kraus A. L., Ireland M. J., Huber D., Mann A. W., Dupuy T. J., 2016, ArXiv e-prints

Lada C. J., Lombardi M., Alves J. F., 2009, ApJ, 703, 52

Le Blanc T. S., Covey K. R., Stassun K. G., 2011, AJ, 142, 55

Liu Y., Joergens V., Bayo A., Nielbock M., Wang H., 2015, A\&A, 582, A22

Loinard L., Torres R. M., Mioduszewski A. J., Rodríguez L. F., 2008, in IAU Symposium, Vol. 248, IAU Symposium, Jin W. J., Platais I., Perryman M. A. C., eds., pp. 186-189

Lubow S. H., Artymowicz P., 2000, Protostars and Planets IV, 731 Lubow S. H., Seibert M., Artymowicz P., 1999, ApJ, 526, 1001

Luhman K. L., Mamajek E. E., Allen P. R., Cruz K. L., 2009, ApJ, 703, 399

Luhman K. L., Rieke G. H., Lada C. J., Lada E. A., 1998, ApJ, 508, 347

Luhman K. L., Stauffer J. R., Muench A. A., Rieke G. H., Lada E. A., Bouvier J., Lada C. J., 2003, ApJ, 593, 1093

Mamajek E. E., 2008, Astronomische Nachrichten, 329, 10

Martinache F., 2011, in Society of Photo-Optical Instrumentation Engineers (SPIE) Conference Series, Vol. 8151, Society of Photo-Optical Instrumentation Engineers (SPIE) Conference Series

Mathis J. S., 1990, in Astronomical Society of the Pacific Conference Series, Vol. 12, The Evolution of the Interstellar Medium, Blitz L., ed., pp. 63-77

Meeus J., 1992, Journal of the British Astronomical Association, 
102,109

Metchev S. A., Hillenbrand L. A., 2009, ApJS, 181, 62

Muzerolle J., Allen L. E., Megeath S. T., Hernández J., Gutermuth R. A., 2010, ApJ, 708, 1107

Najita J. R., Andrews S. M., Muzerolle J., 2015, MNRAS, 450, 3559

Nomura H. et al., 2016, ApJL, 819, L7

Olofsson J., Szûcs L., Henning T., Linz H., Pascucci I., Joergens V., 2013, A\&A, 560, A100

Orellana M., Cieza L. A., Schreiber M. R., Merín B., Brown J. M., Pellizza L. J., Romero G. A., 2012, A\&A, 539, A41

Pascucci I., Apai D., Hardegree-Ullman E. E., Kim J. S., Meyer M. R., Bouwman J., 2008, ApJ, 673, 477

Pecaut M. J., Mamajek E. E., 2013, ApJS, 208, 9

Pinilla P., Benisty M., Birnstiel T., 2012, A\&A, 545, A81

Pollack J. B., Hubickyj O., Bodenheimer P., Lissauer J. J., Podolak M., Greenzweig Y., 1996, Icarus, 124, 62

Pott J.-U., Perrin M. D., Furlan E., Ghez A. M., Herbst T. M., Metchev S., 2010, ApJ, 710, 265

Pravdo S. H., Shaklan S. B., Wiktorowicz S. J., Kulkarni S., Lloyd J. P., Martinache F., Tuthill P. G., Ireland M. J., 2006, ApJ, 649, 389

Raghavan D. et al., 2010, ApJS, 190, 1

Rapson V. A., Kastner J. H., Millar-Blanchaer M. A., Dong R., 2015, ArXiv e-prints

Rebollido I. et al., 2015, ArXiv e-prints

Rebull L. M. et al., 2010, ApJS, 186, 259

Rice W. K. M., Armitage P. J., Wood K., Lodato G., 2006a, MNRAS, 373, 1619

Rice W. K. M., Lodato G., Pringle J. E., Armitage P. J., Bonnell I. A., 2006b, MNRAS, 372, L9

Rosotti G. P., Ercolano B., Owen J. E., 2015, ArXiv e-prints

Scholz R.-D. et al., 1999, A\&AS, 137, 305

Sicilia-Aguilar A. et al., 2006, ApJ, 638, 897

Siess L., 2001, in Astronomical Society of the Pacific Conference Series, Vol. 243, From Darkness to Light: Origin and Evolution of Young Stellar Clusters, Montmerle T., André P., eds., p. 581

Simon M. et al., 1995, ApJ, 443, 625

Skrutskie M. F. et al., 2006, AJ, 131, 1163

Strom K. M., Strom S. E., Edwards S., Cabrit S., Skrutskie M. F., 1989, AJ, 97, 1451

Sun K., Kramer C., Ossenkopf V., Bensch F., Stutzki J., Miller M., 2006, A\&A, 451, 539

Vacca W. D., Sheehy C. D., Graham J. R., 2007, ApJ, 662, 272

van der Marel N., van Dishoeck E. F., Bruderer S., Andrews S. M., Pontoppidan K. M., Herczeg G. J., van Kempen T., Miotello A., 2016, A\&A, 585, A58

van der Marel N., van Dishoeck E. F., Bruderer S., Pérez L., Isella A., 2015, A\&A, 579, A106

Wilking B. A., Gagné M., Allen L. E., 2008, Star Formation in the $\rho$ Ophiuchi Molecular Cloud, Reipurth B., ed., p. 351

Yelda S., Lu J. R., Ghez A. M., Clarkson W., Anderson J., Do T., Matthews K., 2010, ApJ, 725, 331 